\title{
On sections of hyperelliptic Lefschetz fibrations
}

\author{
SHUNSUKE TANAKA
}

\begin{abstract}
We construct a relation among right-handed Dehn twists in the mapping class group of a compact oriented surface of genus $g$ with $4 g+4$ boundary components. This relation gives an explicit topological description of $4 g+4$ disjoint $(-1)$-sections of a hyperelliptic Lefschetz fibration of genus $g$ on the manifold $\mathbb{C P}^{2} \#(4 g+5) \overline{\mathbb{C P}}^{2}$.
\end{abstract}

57N13; 20F34

\section{Introduction}

Lefschetz fibrations relate the topology of symplectic 4-manifolds to the combinatorics on relations in Dehn twist generators of mapping class groups of surfaces. It is wellknown that a Lefschetz fibration of genus 1 on the manifold $E(1)=\mathbb{C P}^{2} \# 9 \overline{\mathbb{C P}}^{2}$ constructed by blowing up nine intersections of two generic cubics in $\mathbb{C} \mathbb{P}^{2}$ has twelve singular fibers and nine disjoint ( $(-1)$-sections. Korkmaz and Ozbagci [7] constructed a relation among right-handed Dehn twists in the mapping class group of a torus with nine boundary components to locate a set of nine disjoint $(-1)$-sections in a Kirby diagram of $E(1)$. It is also known to algebraic geometers that a hyperelliptic Lefschetz fibration of genus $g$ on the manifold $X_{g}=\mathbb{C P}^{2} \#(4 g+5) \overline{\mathbb{C P}}^{2}$ has $8 g+4$ singular fibers and $4 g+4$ disjoint $(-1)-$ sections for $g \geq 2$ (see Saitō and Sakakibara [10, Section 3] and Kitagawa and Konno [6, Remark 1.1]).

In this paper we construct a relation among right-handed Dehn twists in the mapping class group of a compact oriented surface of genus $g$ with $4 g+4$ boundary components to locate a set of $4 g+4$ disjoint $(-1)$-sections in a Kirby diagram of $X_{g}$. In the case $g=2$, our relation can be considered as an improvement of Onaran's relations [9] in mapping class groups of surfaces of genus two with at most eight boundary components.

In Section 2 we review basic relations in mapping class groups and produce two relations on a torus with eight boundary components. Combining these relations, we construct a new relation on a surface of genus $g$ with $4 g+4$ boundary components in Section 3. In Section 4 we apply the relation to visualize $4 g+4$ disjoint $(-1)-$ sections in a Kirby diagram of a hyperelliptic Lefschetz fibration of genus $g$. 


\section{Building blocks}

In this section we review basic relations in mapping class groups and produce two relations on a torus with boundary both used in the next section.

\subsection{Basic relations in mapping class groups}

Let $\Sigma_{g, r}$ be a compact oriented surface of genus $g$ with $r$ boundary components and Diff $+\Sigma_{g, r}$ the group of orientation-preserving diffeomorphisms of $\Sigma_{g, r}$ fixing the boundary $\partial \Sigma_{g, r}$ pointwise equipped with the $C^{\infty}$-topology. The group $\pi_{0}\left(\right.$ Diff $\left._{+} \Sigma_{g, r}\right)$ of path-components of Diff $+\Sigma_{g, r}$ is called the mapping class group of $\Sigma_{g, r}$ and we denote it by $\mathcal{M}_{g, r}$. We denote by $\mathcal{F}_{g, r}$ the free group generated by all isotopy classes $\mathcal{S}_{g, r}$ of simple closed curves in the interior of $\Sigma_{g, r}$. There is a natural epimorphism $\varpi: \mathcal{F}_{g, r} \rightarrow \mathcal{M}_{g, r}$ which sends (the isotopy class of) a simple closed curve $a$ in the interior of $\Sigma_{g, r}$ to the right-handed Dehn twist $t_{a}$ along $a$. We set $\mathcal{R}_{g, r}:=\operatorname{Ker} \varpi$.

A word in the generators $\mathcal{S}_{g, r}$ is called positive if it includes no negative exponents. We put ${ }_{W}(c):=t_{a_{r}}^{\varepsilon_{r}} \cdots t_{a_{1}}^{\varepsilon_{1}}(c) \in \mathcal{S}_{g, r}$ for $c \in \mathcal{S}_{g, r}$ and $W=a_{r}^{\varepsilon_{r}} \cdots a_{1}^{\varepsilon_{1}} \in \mathcal{F}_{g, r}\left(a_{1}, \ldots, a_{r}\right.$ in $\mathcal{S}_{g, r}, \varepsilon_{1}, \ldots, \varepsilon_{r}$ in $\left.\{ \pm 1\}\right)$. We often denote $a^{-1}$ by $\bar{a}$ for an element $a$ of $\mathcal{S}_{g, r}$. For two words $W_{1}, W_{2} \in \mathcal{F}_{g, r}$, we denote $W_{1} \equiv W_{2}$ if $\varpi\left(W_{1}\right)=\varpi\left(W_{2}\right)$. If the relation $W_{1} \equiv W_{2}$ holds for $W_{1}, W_{2} \in \mathcal{F}_{g, r}$, we obtain another relation $V W_{1} V^{-1} \equiv V W_{2} V^{-1}$, which is called a conjugate of $W_{1} \equiv W_{2}$, for every $V \in \mathcal{F}_{g, r}$.

We recall definitions of basic relations in mapping class groups.

Definition 2.1 [3] (1) For disjoint simple closed curves $a, b$ in the interior of $\Sigma_{g, r}$, we have a relation $a b \equiv b a$ in $\mathcal{F}_{g, r}$ called a commutativity relation. A regular neighborhood of $a \cup b$ is the disjoint union of two annuli.

(2) For simple closed curves $a, b$ in the interior of $\Sigma_{g, r}$ which intersect transversely at one point, we have a relation $a b a \equiv b a b$ in $\mathcal{F}_{g, r}$ called a braid relation. A regular neighborhood of $a \cup b$ is a torus with one boundary component.

(3) For simple closed curves $\alpha, \sigma, \gamma, \delta_{1}, \delta_{2}, \delta_{3}, \delta_{4}$ in the interior of $\Sigma_{g, r}$ shown in Figure 1 , we have a relation $\delta_{1} \delta_{2} \delta_{3} \delta_{4} \equiv \gamma \sigma \alpha$ in $\mathcal{F}_{g, r}$ called a lantern relation. The union of $\delta_{1}, \delta_{2}, \delta_{3}, \delta_{4}$ bounds a sphere with four boundary components in $\Sigma_{g, r}$.

(4) An ordered $n$-tuple $\left(c_{1}, \ldots, c_{n}\right)$ of simple closed curves in the interior of $\Sigma_{g, r}$ is called a chain of length $n$ if $c_{i}$ and $c_{i+1}$ intersect transversely at one point ( $i=$ $1, \ldots, n-1)$ and other $c_{i}$ and $c_{j}$ never intersect. For a chain $\left(c_{1}, \ldots, c_{2 g+1}\right)$ of length $2 g+1$ on $\Sigma_{g, 0}$, we have a relation $\left(c_{1} \cdots c_{2 g+1} c_{2 g+1} \cdots c_{1}\right)^{2} \equiv 1$ in $\mathcal{F}_{g, 0}$ called a hyperelliptic relation (see Figure 2). 


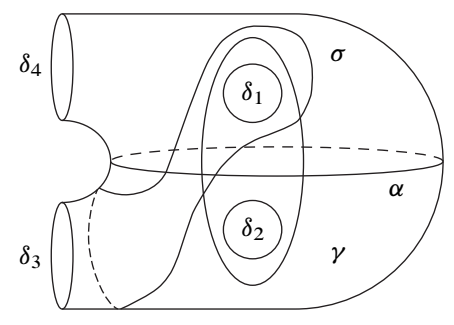

Figure 1: Lantern relation

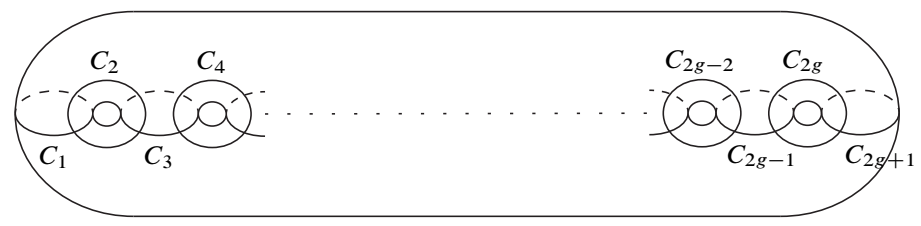

Figure 2: Hyperelliptic relation

Remark 2.2 Let $a$ and $b$ be simple closed curves in the interior of $\Sigma_{\underline{g}, r}$ and $c$ the simple closed curve $t_{b}(a)={ }_{b}(a)$. Then we have the relation $c \equiv b a \bar{b}$ in $\mathcal{F}_{g, r}$. If $a$ and $b$ intersect transversely at one point, we have another relation $b \equiv a c \bar{a}$. This relation together with the relation $c \equiv b a \bar{b}$ yields a braid relation $a b a \equiv b a b$.

\subsection{Two relations on a torus with boundary}

In this subsection we construct two relations on a torus with eight boundary components. The first relation is the following.

Proposition 2.3 Relation (A) For simple closed curves in the interior of $\Sigma_{1,8}$ shown in Figure 3, we have the relation

$$
a_{1} a_{2} \delta_{1} \delta_{2} \delta_{3} \delta_{4} \delta_{5} \delta_{6} \equiv a_{5} a_{4} b_{2} \bar{a}_{4} \sigma_{1} \sigma_{4} a_{10} a_{3} b_{2} \bar{a}_{3} \sigma_{2} a_{5} a_{3} a_{8} b_{2} \bar{a}_{8} \bar{a}_{3} \sigma_{3} \sigma_{5} a_{11} .
$$

We make use of the five-holed torus relation found by Korkmaz and Ozbagci [7] in order to prove Proposition 2.3.

Lemma 2.4 (Korkmaz-Ozbagci [7]) For simple closed curves in the interior of $\Sigma_{1,5}$ shown on the right in Figure 4, we have the relation

$$
\delta_{2} \delta_{1} a_{2} \gamma \delta_{3} \equiv a_{5} b_{2} a_{3} a_{4} a_{5} b_{2} \sigma_{1} a_{6} a_{3} b_{2} \sigma_{2} a_{8} .
$$




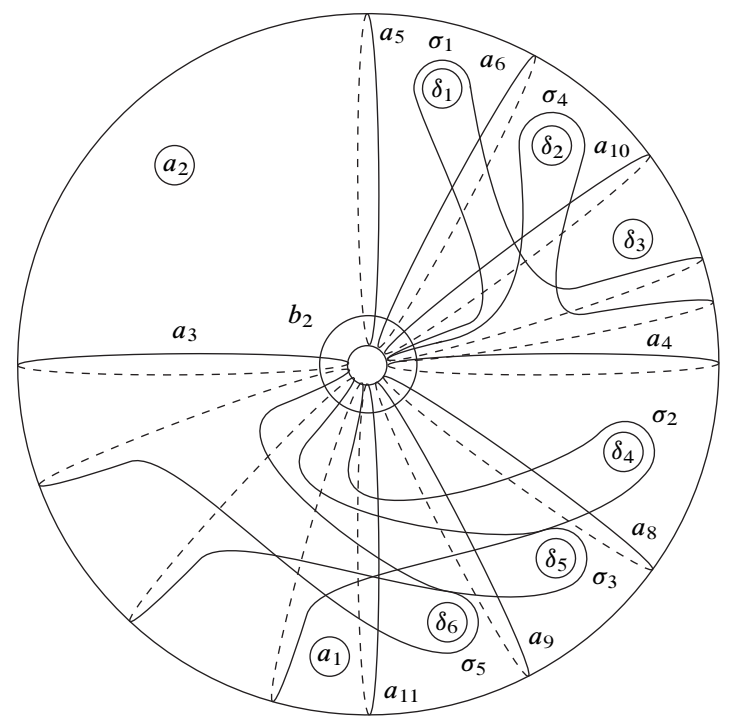

Figure 3: Relation (A)

Remark 2.5 The relation in Lemma 2.4 is deduced from the original five-holed torus relation

$$
\delta_{2} \delta_{1} a_{2} \gamma \delta_{3} \equiv a_{5} a_{3} a_{4} b_{2} \sigma_{1} a_{6} a_{3} b_{2} \sigma_{2} a_{8} a_{5} b_{2}
$$

(see [7, Section 3.5]) by using commutativity relations and conjugations.
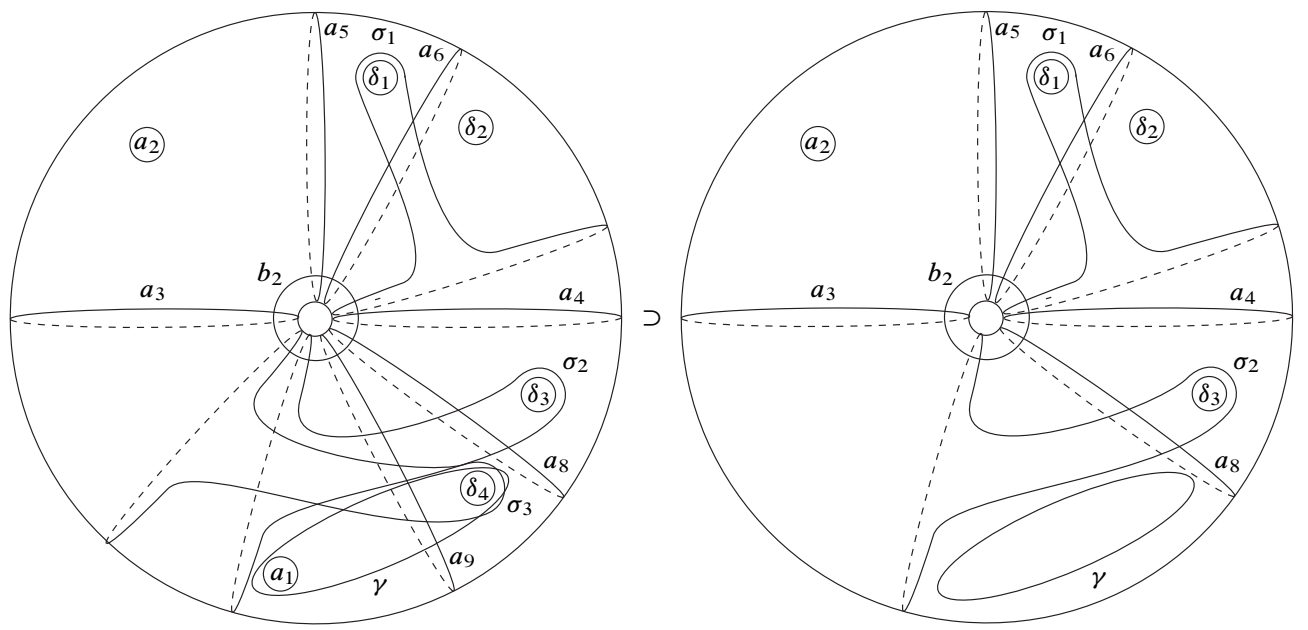

Figure 4: Five-holed torus relation 
Proof of Proposition 2.3 Applying commutativity relations and conjugations to the five-holed torus relation in Lemma 2.4, we obtain

$$
\begin{aligned}
a_{2} \delta_{1} \delta_{2} \delta_{3} \gamma \equiv a_{5} b_{2} a_{3} a_{4} a_{5} b_{2} \sigma_{1} a_{6} a_{3} b_{2} \sigma_{2} a_{8} & \equiv a_{8} b_{2} \bar{a}_{8} a_{8} a_{3} a_{4} a_{5} b_{2} \sigma_{1} a_{6} a_{3} b_{2} \sigma_{2} a_{5} \\
& \equiv a_{8} a_{3} a_{4} a_{5} b_{2} \sigma_{1} a_{6} a_{3} b_{2} \sigma_{2} a_{5} a_{8} b_{2} \bar{a}_{8} .
\end{aligned}
$$

Multiplying both sides of this relation by $\bar{\gamma}$, we have

$$
a_{2} \delta_{1} \delta_{2} \delta_{3} \equiv a_{8} a_{3} a_{4} a_{5} b_{2} \sigma_{1} a_{6} a_{3} b_{2} \sigma_{2} a_{5} a_{8} b_{2} \bar{a}_{8} \bar{\gamma} .
$$

We embed $\Sigma_{1,5}$ into $\Sigma_{1,6}$ and take simple closed curves $a_{1}, a_{9}, \delta_{4}, \sigma_{3}$ in the interior of $\Sigma_{1,6}$ shown in Figure 4. Then we have a lantern relation

$$
\delta_{4} a_{1} a_{3} a_{8} \equiv \gamma \sigma_{3} a_{9} .
$$

Combining these relations and applying commutativity relations, we obtain

$$
\begin{aligned}
a_{8} a_{3} a_{1} a_{2} \delta_{1} \delta_{2} \delta_{3} \delta_{4} & \equiv a_{8} a_{3} a_{4} a_{5} b_{2} \sigma_{1} a_{6} a_{3} b_{2} \sigma_{2} a_{5} a_{8} b_{2} \bar{a}_{8} \bar{\gamma} \gamma \sigma_{3} a_{9} \\
& \equiv a_{8} a_{3} a_{4} a_{5} b_{2} \sigma_{1} a_{6} a_{3} b_{2} \sigma_{2} a_{5} a_{8} b_{2} \bar{a}_{8} \sigma_{3} a_{9} .
\end{aligned}
$$

Multiplying both sides of this relation by $\bar{a}_{3} \bar{a}_{8}$, we have a relation

$$
a_{1} a_{2} \delta_{1} \delta_{2} \delta_{3} \delta_{4} \equiv a_{4} a_{5} b_{2} \sigma_{1} a_{6} a_{3} b_{2} \sigma_{2} a_{5} a_{8} b_{2} \bar{a}_{8} \sigma_{3} a_{9}
$$

on $\Sigma_{1,6}$.

We change the name $\delta_{2}$ of a curve in relation (A1) into $\gamma$ (shown on the right in Figure 5) and apply commutativity relations and conjugations to it to obtain

$$
\begin{aligned}
a_{1} a_{2} \delta_{1} \delta_{3} \delta_{4} \gamma & \equiv a_{4} a_{5} b_{2} \sigma_{1} a_{6} a_{3} b_{2} \sigma_{2} a_{5} a_{8} b_{2} \bar{a}_{8} \sigma_{3} a_{9} \\
& \equiv a_{5} a_{4} b_{2} \bar{a}_{4} a_{4} \sigma_{1} a_{6} a_{3} b_{2} \sigma_{2} a_{5} a_{8} b_{2} \bar{a}_{8} \sigma_{3} a_{9} \\
& \equiv a_{4} a_{6} a_{3} b_{2} \sigma_{2} a_{5} a_{8} b_{2} \bar{a}_{8} \sigma_{3} a_{9} a_{5} a_{4} b_{2} \bar{a}_{4} \sigma_{1} .
\end{aligned}
$$

Multiplying both sides of this relation by $\bar{\gamma}$, we have

$$
a_{1} a_{2} \delta_{1} \delta_{3} \delta_{4} \equiv a_{4} a_{6} a_{3} b_{2} \sigma_{2} a_{5} a_{8} b_{2} \bar{a}_{8} \sigma_{3} a_{9} a_{5} a_{4} b_{2} \bar{a}_{4} \sigma_{1} \bar{\gamma} .
$$

We embed $\Sigma_{1,6}$ into $\Sigma_{1,7}$ and take simple closed curves $a_{10}, \delta_{2}, \delta_{5}, \sigma_{4}$ in the interior of $\Sigma_{1,7}$ shown in Figure 5. Then we have a lantern relation

$$
\delta_{2} \delta_{5} a_{4} a_{6} \equiv \gamma \sigma_{4} a_{10} .
$$

Combining these relations and applying commutativity relations, we obtain

$$
\begin{aligned}
a_{4} a_{6} a_{1} a_{2} \delta_{1} \delta_{2} \delta_{3} \delta_{4} \delta_{5} & \equiv a_{4} a_{6} a_{3} b_{2} \sigma_{2} a_{5} a_{8} b_{2} \bar{a}_{8} \sigma_{3} a_{9} a_{5} a_{4} b_{2} \bar{a}_{4} \sigma_{1} \bar{\gamma} \gamma \sigma_{4} a_{10} \\
& \equiv a_{4} a_{6} a_{3} b_{2} \sigma_{2} a_{5} a_{8} b_{2} \bar{a}_{8} \sigma_{3} a_{9} a_{5} a_{4} b_{2} \bar{a}_{4} \sigma_{1} \sigma_{4} a_{10} .
\end{aligned}
$$




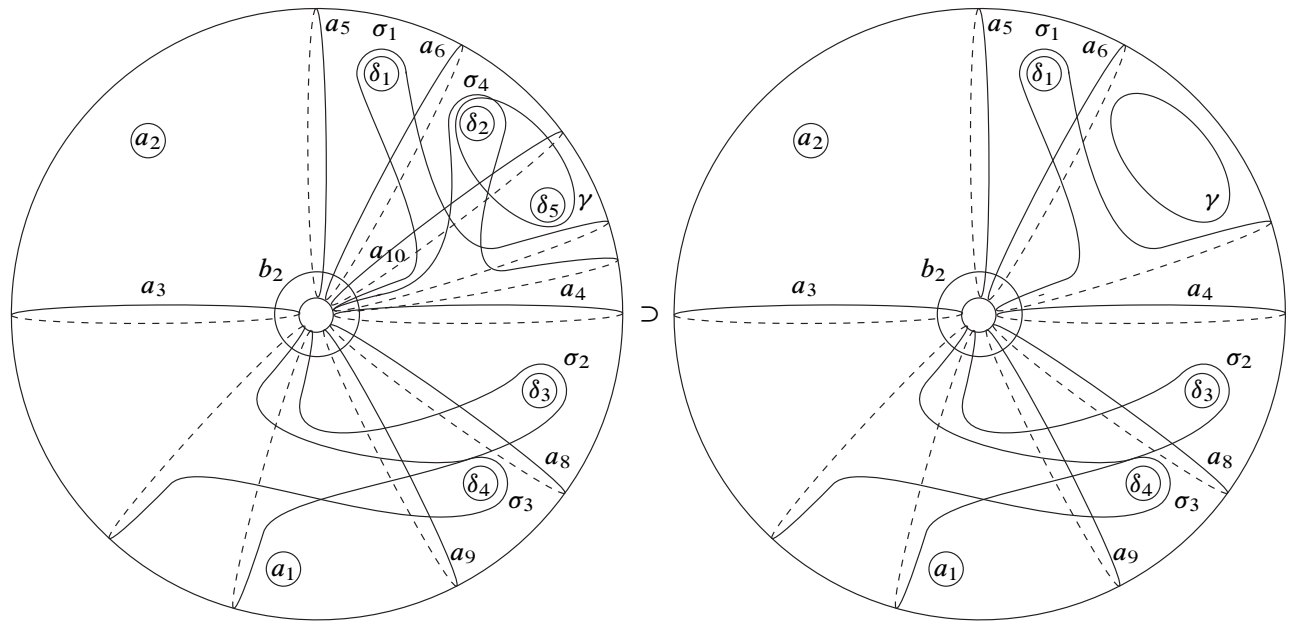

Figure 5: Embedding of $\Sigma_{1,6}$ into $\Sigma_{1,7}$ (I)

Multiplying both sides of this relation by $\bar{a}_{6} \bar{a}_{4}$, we have a relation

$$
a_{1} a_{2} \delta_{1} \delta_{2} \delta_{3} \delta_{4} \delta_{5} \equiv a_{3} b_{2} \sigma_{2} a_{5} a_{8} b_{2} \bar{a}_{8} \sigma_{3} a_{9} a_{5} a_{4} b_{2} \bar{a}_{4} \sigma_{1} \sigma_{4} a_{10}
$$

on $\Sigma_{1,7}$.

We change the name $a_{1}$ of a curve in relation (A2) into $\gamma$ (shown on the right in Figure 6) and apply commutativity relations and conjugations to it to obtain

$$
\begin{aligned}
a_{2} \delta_{1} \delta_{2} \delta_{3} \delta_{4} \delta_{5} \gamma & \equiv a_{3} b_{2} \sigma_{2} a_{5} a_{8} b_{2} \bar{a}_{8} \sigma_{3} a_{9} a_{5} a_{4} b_{2} \bar{a}_{4} \sigma_{1} \sigma_{4} a_{10} \\
& \equiv a_{3} b_{2} \bar{a}_{3} \sigma_{2} a_{5} a_{3} a_{8} b_{2} \bar{a}_{8} \sigma_{3} a_{9} a_{5} a_{4} b_{2} \bar{a}_{4} \sigma_{1} \sigma_{4} a_{10} \\
& \equiv a_{3} b_{2} \bar{a}_{3} \sigma_{2} a_{5} a_{3} a_{8} b_{2} \bar{a}_{8} \bar{a}_{3} \sigma_{3} a_{3} a_{9} a_{5} a_{4} b_{2} \bar{a}_{4} \sigma_{1} \sigma_{4} a_{10} \\
& \equiv a_{3} a_{9} a_{5} a_{4} b_{2} \bar{a}_{4} \sigma_{1} \sigma_{4} a_{10} a_{3} b_{2} \bar{a}_{3} \sigma_{2} a_{5} a_{3} a_{8} b_{2} \bar{a}_{8} \bar{a}_{3} \sigma_{3} .
\end{aligned}
$$

Multiplying both sides of this relation by $\bar{\gamma}$, we have

$$
a_{2} \delta_{1} \delta_{2} \delta_{3} \delta_{4} \delta_{5} \equiv a_{3} a_{9} a_{5} a_{4} b_{2} \bar{a}_{4} \sigma_{1} \sigma_{4} a_{10} a_{3} b_{2} \bar{a}_{3} \sigma_{2} a_{5} a_{3} a_{8} b_{2} \bar{a}_{8} \bar{a}_{3} \sigma_{3} \bar{\gamma} \text {. }
$$

We embed $\Sigma_{1,7}$ into $\Sigma_{1,8}$ and take simple closed curves $a_{1}, a_{11}, \delta_{6}, \sigma_{5}$ in the interior of $\Sigma_{1,8}$ shown in Figure 6. Then we have a lantern relation

$$
\delta_{6} a_{1} a_{3} a_{9} \equiv \gamma \sigma_{5} a_{11} .
$$

Combining these relations and applying commutativity relations, we obtain

$$
\begin{aligned}
a_{3} a_{9} a_{1} a_{2} \delta_{1} \delta_{2} \delta_{3} \delta_{4} \delta_{5} \delta_{6} & \equiv a_{3} a_{9} a_{5} a_{4} b_{2} \bar{a}_{4} \sigma_{1} \sigma_{4} a_{10} a_{3} b_{2} \bar{a}_{3} \sigma_{2} a_{5} a_{3} a_{8} b_{2} \bar{a}_{8} \bar{a}_{3} \sigma_{3} \bar{\gamma} \gamma \sigma_{5} a_{11} \\
& \equiv a_{3} a_{9} a_{5} a_{4} b_{2} \bar{a}_{4} \sigma_{1} \sigma_{4} a_{10} a_{3} b_{2} \bar{a}_{3} \sigma_{2} a_{5} a_{3} a_{8} b_{2} \bar{a}_{8} \bar{a}_{3} \sigma_{3} \sigma_{5} a_{11} .
\end{aligned}
$$



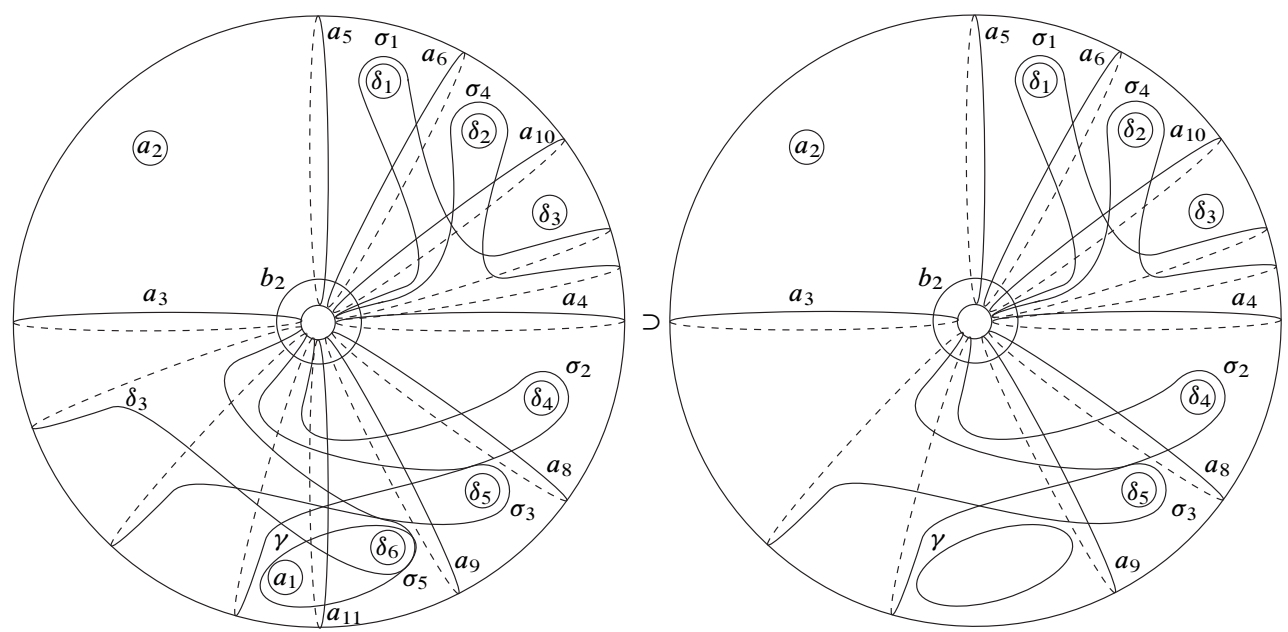

Figure 6: Embedding of $\Sigma_{1,7}$ into $\Sigma_{1,8}$ (I)

Multiplying both sides of this relation by $\bar{a}_{9} \bar{a}_{3}$, we finally obtain relation (A). This completes the proof of Proposition 2.3.

The second relation constructed in this subsection is the following.

Proposition 2.6 Relation (B) For simple closed curves in the interior of $\Sigma_{1,8}$ shown in Figure 7, we have the relation

$$
a_{1} a_{2} a_{7} a_{8} \delta_{1} \delta_{2} \delta_{3} \delta_{4} \equiv a_{4} a_{5}^{\prime \prime} \bar{a}_{6} b_{2} a_{6} a_{3} b_{2} \bar{a}_{3} \tau^{\prime} \tau^{\prime \prime \prime} a_{5} a_{4}^{\prime \prime} \bar{a}_{3} b_{2} a_{3} a_{6} b_{2} \bar{a}_{6} \tau \tau^{\prime \prime} .
$$

We make use of the four-holed torus relation found by Korkmaz and Ozbagci [7] in order to prove Proposition 2.6.

Lemma 2.7 (Korkmaz-Ozbagci [7]) For simple closed curves in the interior of $\Sigma_{1,4}$ shown on the left in Figure 8, we have the relation

$$
a_{2} a_{1} a_{7} \gamma \equiv\left(a_{3} a_{6} b_{2} a_{4} a_{5} b_{2}\right)^{2} .
$$

Remark 2.8 The relation in Lemma 2.7 is not the exact four-holed torus relation but the relation written in a more symmetric form (see [7, Section 3.4, Remark]).

Proof of Proposition 2.6 We consider the four-holed torus relation reviewed in Lemma 2.7. We then embed $\Sigma_{1,4}$ into $\Sigma_{1,5}$ and take simple closed curves $a_{5}^{\prime}, a_{8}, \delta_{1}, \tau$ in the interior of $\Sigma_{1,5}$ shown in Figure 8. Then we have a lantern relation

$$
\delta_{1} a_{8} a_{6} a_{5} \equiv \gamma \tau a_{5}^{\prime} .
$$




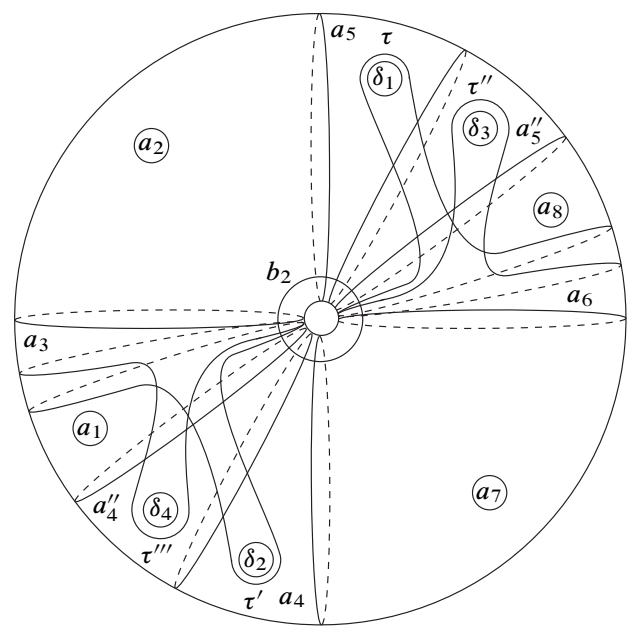

Figure 7: Relation (B)

Combining this relations with the four-holed torus relation, and applying commutativity relations and conjugations, we obtain a relation

(B1)

$$
\begin{aligned}
a_{1} a_{2} a_{7} a_{8} \delta_{1} & \equiv \bar{a}_{5} a_{4} a_{5} b_{2} a_{3} a_{6} b_{2} a_{4} a_{5} b_{2} a_{3} a_{6} b_{2} \bar{\gamma} \cdot \bar{a}_{6} \gamma \tau a_{5}^{\prime} \\
& \equiv a_{4} b_{2} a_{3} a_{6} b_{2} a_{4} a_{5} b_{2} a_{6} a_{3} b_{2} \bar{a}_{6} \tau a_{5}^{\prime} \\
& \equiv a_{4} a_{5} b_{2} a_{6} a_{3} b_{2} \bar{a}_{6} \tau a_{5}^{\prime} a_{4} b_{2} a_{3} a_{6} b_{2}
\end{aligned}
$$
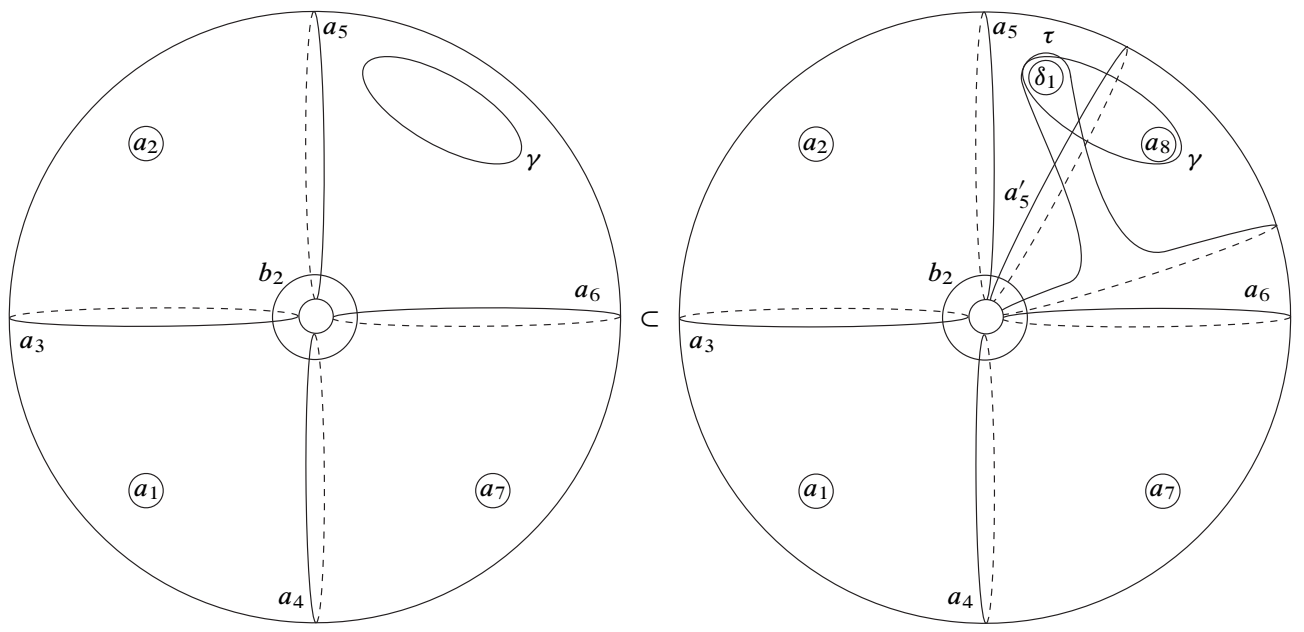

Figure 8: Four-holed torus relation 
on $\Sigma_{1,5}$.
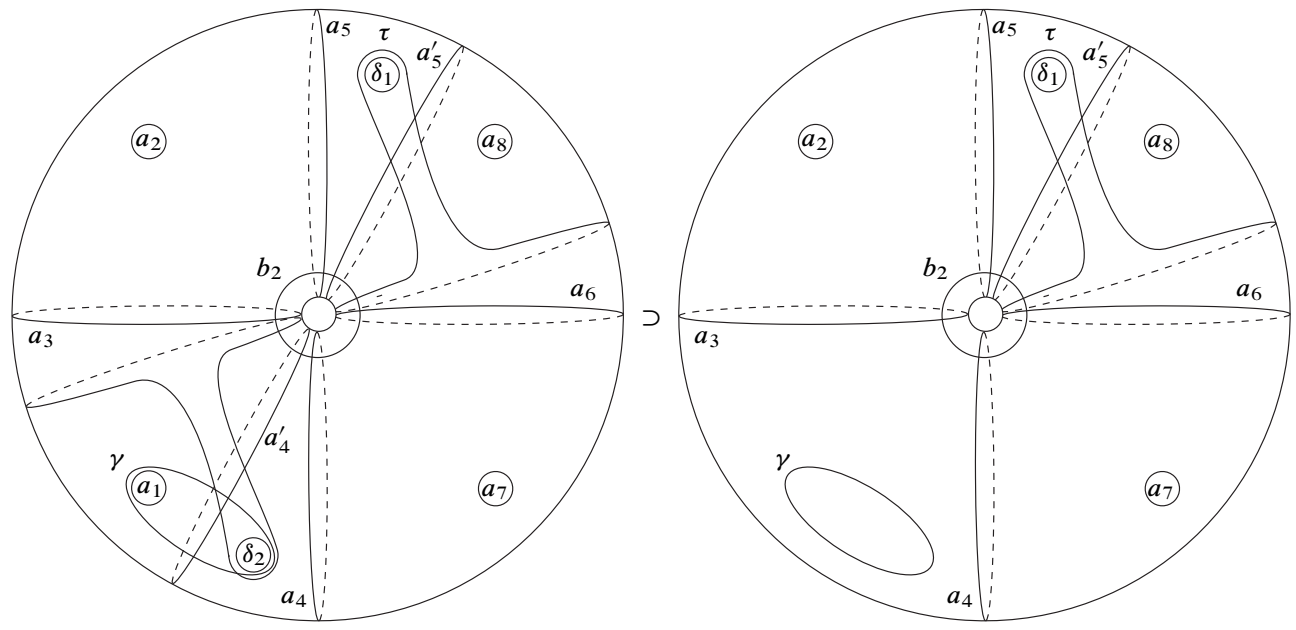

Figure 9: Embedding of $\Sigma_{1,5}$ into $\Sigma_{1,6}$

We change the name $a_{1}$ of a curve in relation (B1) into $\gamma$ (shown on the right in Figure 9) to obtain

$$
\gamma a_{2} a_{7} a_{8} \delta_{1} \equiv a_{4} a_{5} b_{2} a_{6} a_{3} b_{2} \bar{a}_{6} \tau a_{5}^{\prime} a_{4} b_{2} a_{3} a_{6} b_{2} .
$$

We embed $\Sigma_{1,5}$ into $\Sigma_{1,6}$ and take simple closed curves $a_{1}, a_{4}^{\prime}, \delta_{2}, \tau^{\prime}$ in the interior of $\Sigma_{1,6}$ shown in Figure 9. Then we have a lantern relation

$$
a_{4} a_{3} a_{1} \delta_{2} \equiv \gamma \tau^{\prime} a_{4}^{\prime}
$$

Combining these relations and applying commutativity relations and conjugations, we obtain a relation

$$
\begin{aligned}
a_{1} a_{2} a_{7} a_{8} \delta_{1} \delta_{2} & \equiv \bar{a}_{4} a_{4} a_{5} b_{2} a_{6} a_{3} b_{2} \bar{a}_{6} \tau a_{5}^{\prime} a_{4} b_{2} a_{3} a_{6} b_{2} \bar{\gamma} \cdot \bar{a}_{3} \gamma \tau^{\prime} a_{4}^{\prime} \\
& \equiv a_{5} b_{2} a_{6} a_{3} b_{2} \bar{a}_{6} \tau a_{5}^{\prime} a_{4} b_{2} a_{6} a_{3} b_{2} \bar{a}_{3} \tau^{\prime} a_{4}^{\prime} \\
& \equiv b_{2} a_{6} a_{3} b_{2} \bar{a}_{3} \tau^{\prime} a_{4}^{\prime} a_{5} b_{2} a_{6} a_{3} b_{2} \bar{a}_{6} \tau a_{5}^{\prime} a_{4} .
\end{aligned}
$$

on $\Sigma_{1,6}$.

We change the name $a_{8}$ of a curve in relation (B2) into $\gamma$ (shown on the left in Figure 10) to obtain

$$
a_{1} a_{2} a_{7} \gamma \delta_{1} \delta_{2} \equiv b_{2} a_{6} a_{3} b_{2} \bar{a}_{3} \tau^{\prime} a_{4}^{\prime} a_{5} b_{2} a_{6} a_{3} b_{2} \bar{a}_{6} \tau a_{5}^{\prime} a_{4} .
$$



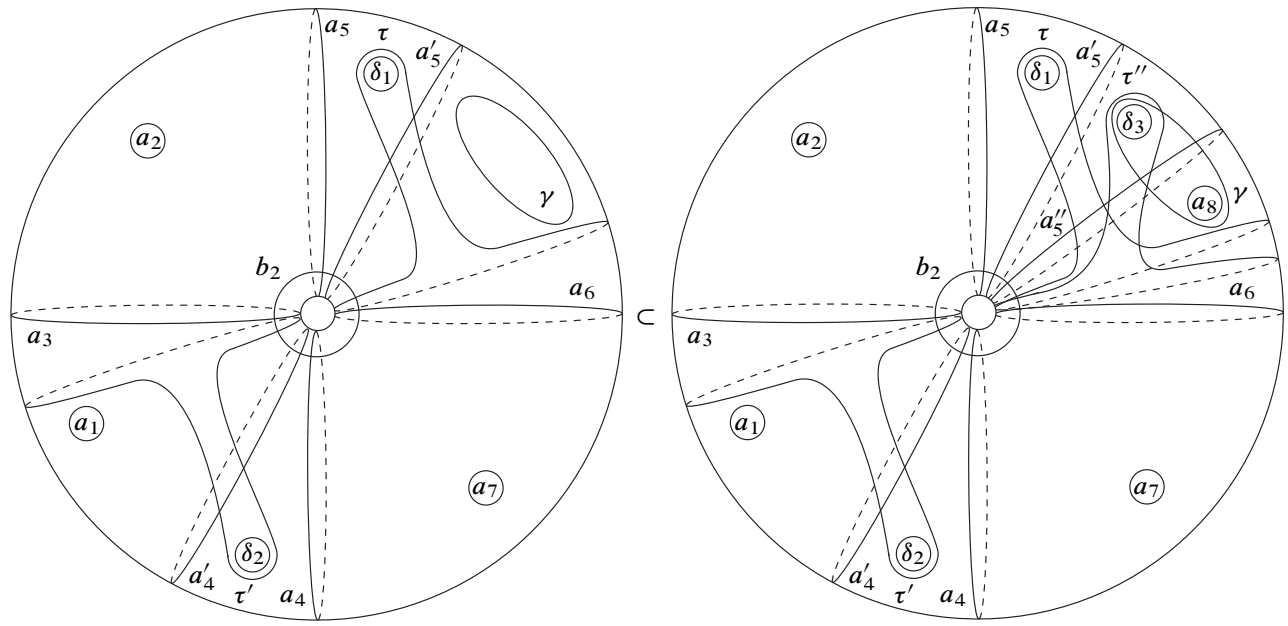

Figure 10: Embedding of $\Sigma_{1,6}$ into $\Sigma_{1,7}$ (II)

We embed $\Sigma_{1,6}$ into $\Sigma_{1,7}$ and take simple closed curves $a_{5}^{\prime \prime}, a_{8}, \delta_{3}, \tau^{\prime \prime}$ in the interior of $\Sigma_{1,7}$ shown in Figure 10. Then we have a lantern relation

$$
\delta_{3} a_{8} a_{6} a_{5}^{\prime} \equiv \gamma \tau^{\prime \prime} a_{5}^{\prime \prime}
$$

Combining these relations and applying commutativity relations and conjugations, we obtain a relation

$$
\begin{aligned}
a_{1} a_{2} a_{7} a_{8} \delta_{1} \delta_{2} \delta_{3} & \equiv \bar{a}_{6} b_{2} a_{6} a_{3} b_{2} \bar{a}_{3} \tau^{\prime} a_{4}^{\prime} a_{5} b_{2} a_{6} a_{3} b_{2} \bar{a}_{6} \tau a_{5}^{\prime} a_{4} \bar{\gamma} \cdot \bar{a}_{5}^{\prime} \gamma \tau^{\prime \prime} a_{5}^{\prime \prime} \\
& \equiv \bar{a}_{6} b_{2} a_{6} a_{3} b_{2} \bar{a}_{3} \tau^{\prime} a_{4}^{\prime} a_{5} b_{2} a_{6} a_{3} b_{2} \bar{a}_{6} \tau \tau^{\prime \prime} a_{4} a_{5}^{\prime \prime} \\
& \equiv b_{2} a_{3} a_{6} b_{2} \bar{a}_{6} \tau \tau^{\prime \prime} a_{4} a_{5}^{\prime \prime} \bar{a}_{6} b_{2} a_{6} a_{3} b_{2} \bar{a}_{3} \tau^{\prime} a_{4}^{\prime} a_{5} .
\end{aligned}
$$

We change the name $a_{1}$ of a curve in relation (B3) into $\gamma$ (shown on the right in Figure 11) to obtain

$$
\gamma a_{2} a_{7} a_{8} \delta_{1} \delta_{2} \delta_{3} \equiv b_{2} a_{3} a_{6} b_{2} \bar{a}_{6} \tau \tau^{\prime \prime} a_{4} a_{5}^{\prime \prime} \bar{a}_{6} b_{2} a_{6} a_{3} b_{2} \bar{a}_{3} \tau^{\prime} a_{4}^{\prime} a_{5}
$$

We embed $\Sigma_{1,7}$ into $\Sigma_{1,8}$ and take simple closed curves $a_{1}, a_{4}^{\prime \prime}, \delta_{4}, \tau^{\prime \prime \prime}$ in the interior of $\Sigma_{1,8}$ shown in Figure 11. Then we have a lantern relation

$$
\delta_{4} a_{1} a_{3} a_{4}^{\prime} \equiv \gamma \tau^{\prime \prime \prime} a_{4}^{\prime \prime}
$$




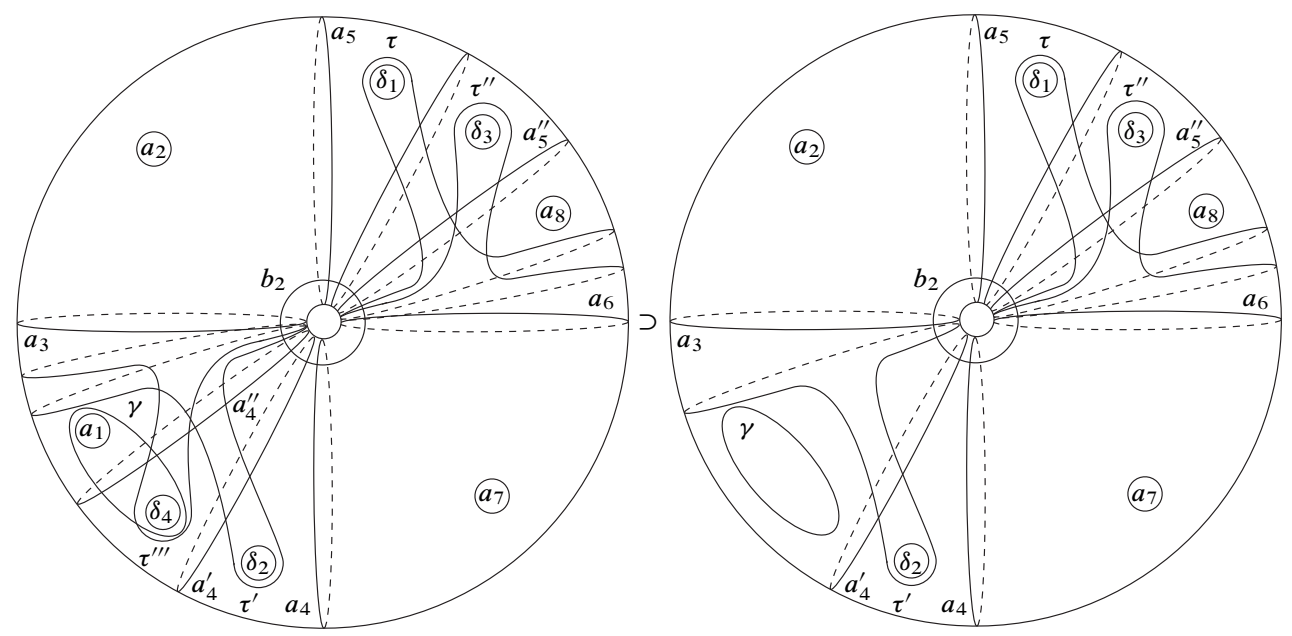

Figure 11: Embedding of $\Sigma_{1,7}$ into $\Sigma_{1,8}$ (II)

Combining these relations and applying commutativity relations and conjugations, we finally obtain relation $(\mathrm{B})$ :

$$
\begin{aligned}
a_{1} a_{2} a_{7} a_{8} \delta_{1} \delta_{2} \delta_{3} \delta_{4} & \equiv \bar{a}_{3} b_{2} a_{3} a_{6} b_{2} \bar{a}_{6} \tau \tau^{\prime \prime} a_{4} a_{5}^{\prime \prime} \bar{a}_{6} b_{2} a_{6} a_{3} b_{2} \bar{a}_{3} \tau^{\prime} a_{4}^{\prime} a_{5} \bar{\gamma} \cdot \bar{a}_{4}^{\prime} \gamma \tau^{\prime \prime \prime} a_{4}^{\prime \prime} \\
& \equiv \bar{a}_{3} b_{2} a_{3} a_{6} b_{2} \bar{a}_{6} \tau \tau^{\prime \prime} a_{4} a_{5}^{\prime \prime} \bar{a}_{6} b_{2} a_{6} a_{3} b_{2} \bar{a}_{3} \tau^{\prime} \tau^{\prime \prime \prime} a_{5} a_{4}^{\prime \prime} \\
& \equiv a_{4} a_{5}^{\prime \prime} \bar{a}_{6} b_{2} a_{6} a_{3} b_{2} \bar{a}_{3} \tau^{\prime} \tau^{\prime \prime \prime} a_{5} a_{4}^{\prime \prime} \bar{a}_{3} b_{2} a_{3} a_{6} b_{2} \bar{a}_{6} \tau \tau^{\prime \prime} .
\end{aligned}
$$

This completes the proof of Proposition 2.6.

Remark 2.9 Both of relations (A) and (B) are different from the eight-holed torus relation of Korkmaz and Ozbagci [7] though the constructions are similar.

\section{Constructions}

In this section we construct a new relation on a compact oriented surface of genus $g$ with $4 g+4$ boundary components by combining copies of relations (A) and (B) obtained in the previous section.

\subsection{Higher genus}

We assume $g \geq 3$. For integers $m, n(0<m \leq n)$ and words $W_{m}, W_{m+1}, \ldots, W_{n}$ in $\mathcal{F}_{g, r}$, we denote the product $W_{m} W_{m+1} \cdots W_{n}$ (respectively $W_{n} \cdots W_{m+1} W_{m}$ ) by $\prod_{i=m}^{n} W_{i}$ (respectively $\prod_{i=n}^{m} W_{i}$ ). 
Theorem 3.1 Relation $\left(\mathrm{H}_{g}\right)$ For simple closed curves in the interior of $\Sigma_{g, 4 g+4}$ shown in Figure 12, we have the relation

$$
\begin{aligned}
\delta_{1} \delta_{2} \cdots \delta_{4 g+3} \delta_{4 g+4} \equiv \prod_{i=g-1}^{2} \beta_{i}^{\prime \prime \prime} & \beta_{i} \tau_{i-1}^{\prime} \tau_{i-1}^{\prime \prime \prime} \cdot \beta_{1} \sigma_{1}^{\prime} \sigma_{4}^{\prime} a_{3 g+3} \beta_{1}^{\prime} \sigma_{2}^{\prime} a_{1} \beta_{1}^{\prime \prime} \sigma_{3}^{\prime} \sigma_{5}^{\prime} \\
& \times \prod_{i=2}^{g-1} \beta_{i}^{\prime \prime} \beta_{i}^{\prime} \tau_{i-1} \tau_{i-1}^{\prime \prime} \cdot \beta_{g} \sigma_{1} \sigma_{4} a_{3 g} \beta_{g}^{\prime} \sigma_{2} a_{3 g-1} \beta_{g}^{\prime \prime} \sigma_{3} \sigma_{5}
\end{aligned}
$$

in $\mathcal{M}_{g, 4 g+4}$, where

$$
\begin{array}{rlll}
\beta_{1}:=a_{3 g+4}\left(b_{1}\right), & \beta_{1}^{\prime}:=a_{3}\left(b_{1}\right), & \beta_{1}^{\prime \prime}:=a_{3 g+5} a_{3}\left(b_{1}\right), \\
\beta_{g}:=a_{3 g+1}\left(b_{g}\right), & \beta_{g}^{\prime}:=a_{3 g-3}\left(b_{g}\right), & \beta_{g}^{\prime \prime}:=a_{3 g-3} a_{3 g+2}\left(b_{g}\right), \\
\beta_{i}:=a_{3 i-3}\left(b_{i}\right), & \beta_{i}^{\prime}:=a_{3 i}\left(b_{i}\right), & \beta_{i}^{\prime \prime}:=\bar{a}_{3 i-3}\left(b_{i}\right), & \beta_{i}^{\prime \prime \prime}:=\bar{a}_{3 i}\left(b_{i}\right),
\end{array}
$$

and $i=2, \ldots, g-1$.

Proof We combine two copies of relation (A) and $g-2$ copies of relation (B) to obtain the desired relation. We first consider two relations for simple closed curves shown in Figure 13. One is a copy of relation (A):

$a_{5} a_{4}^{\prime} \delta_{1} \delta_{4} \delta_{6} \delta_{2} \delta_{3} \delta_{5}$

$$
\equiv a_{1} a_{3 g+4} b_{1} \bar{a}_{3 g+4} \sigma_{1}^{\prime} \sigma_{4}^{\prime} a_{3 g+3} a_{3} b_{1} \bar{a}_{3} \sigma_{2}^{\prime} a_{1} a_{3} a_{3 g+5} b_{1} \bar{a}_{3 g+5} \bar{a}_{3} \sigma_{3}^{\prime} \sigma_{5}^{\prime} a_{2}
$$

Applying commutativity relations and conjugations, we obtain a relation $a_{4}^{\prime} a_{5} \delta_{1} \delta_{2} \delta_{3} \delta_{4} \delta_{5} \delta_{6}$

$$
\begin{aligned}
& \equiv a_{1} a_{2} a_{3 g+4} b_{1} \bar{a}_{3 g+4} \sigma_{1}^{\prime} \sigma_{4}^{\prime} a_{3 g+3} a_{3} b_{1} \bar{a}_{3} \sigma_{2}^{\prime} a_{1} a_{3} a_{3 g+5} b_{1} \bar{a}_{3 g+5} \bar{a}_{3} \sigma_{3}^{\prime} \sigma_{5}^{\prime} \\
& \equiv a_{1} a_{2} \beta_{1} \sigma_{1}^{\prime} \sigma_{4}^{\prime} a_{3 g+3} \beta_{1}^{\prime} \sigma_{2}^{\prime} a_{1} \beta_{1}^{\prime \prime} \sigma_{3}^{\prime} \sigma_{5}^{\prime} .
\end{aligned}
$$

Note that $\beta_{1} \equiv a_{3 g+4} b_{1} \bar{a}_{3 g+4}, \beta_{1}^{\prime} \equiv a_{3} b_{1} \bar{a}_{3}$ and $\beta_{1}^{\prime \prime} \equiv a_{3 g+5} a_{3} b_{1} \bar{a}_{3} \bar{a}_{3 g+5}$ by Remark 2.2. The other is a copy of relation (B):

$$
a_{1} a_{2} a_{7}^{\prime} a_{8} \delta_{7} \delta_{8} \delta_{9} \delta_{10} \equiv a_{4} a_{5}^{\prime} \bar{a}_{6} b_{2} a_{6} a_{3} b_{2} \bar{a}_{3} \tau_{1}^{\prime} \tau_{1}^{\prime \prime \prime} a_{5} a_{4}^{\prime} \bar{a}_{3} b_{2} a_{3} a_{6} b_{2} \bar{a}_{6} \tau_{1} \tau_{1}^{\prime \prime}
$$

Applying commutativity relations and conjugations, we obtain a relation

$$
a_{1} a_{2} a_{7}^{\prime} a_{8} \delta_{7} \delta_{8} \delta_{9} \delta_{10} \equiv a_{5} a_{4}^{\prime} \bar{a}_{3} b_{2} a_{3} a_{6} b_{2} \bar{a}_{6} \tau_{1} \tau_{1}^{\prime \prime} a_{4} a_{5}^{\prime} \bar{a}_{6} b_{2} a_{6} a_{3} b_{2} \bar{a}_{3} \tau_{1}^{\prime} \tau_{1}^{\prime \prime \prime} .
$$

We embed two copies of $\Sigma_{1,8}$ in Figure 13 into $\Sigma_{2,12}$ as shown in Figure 14. 


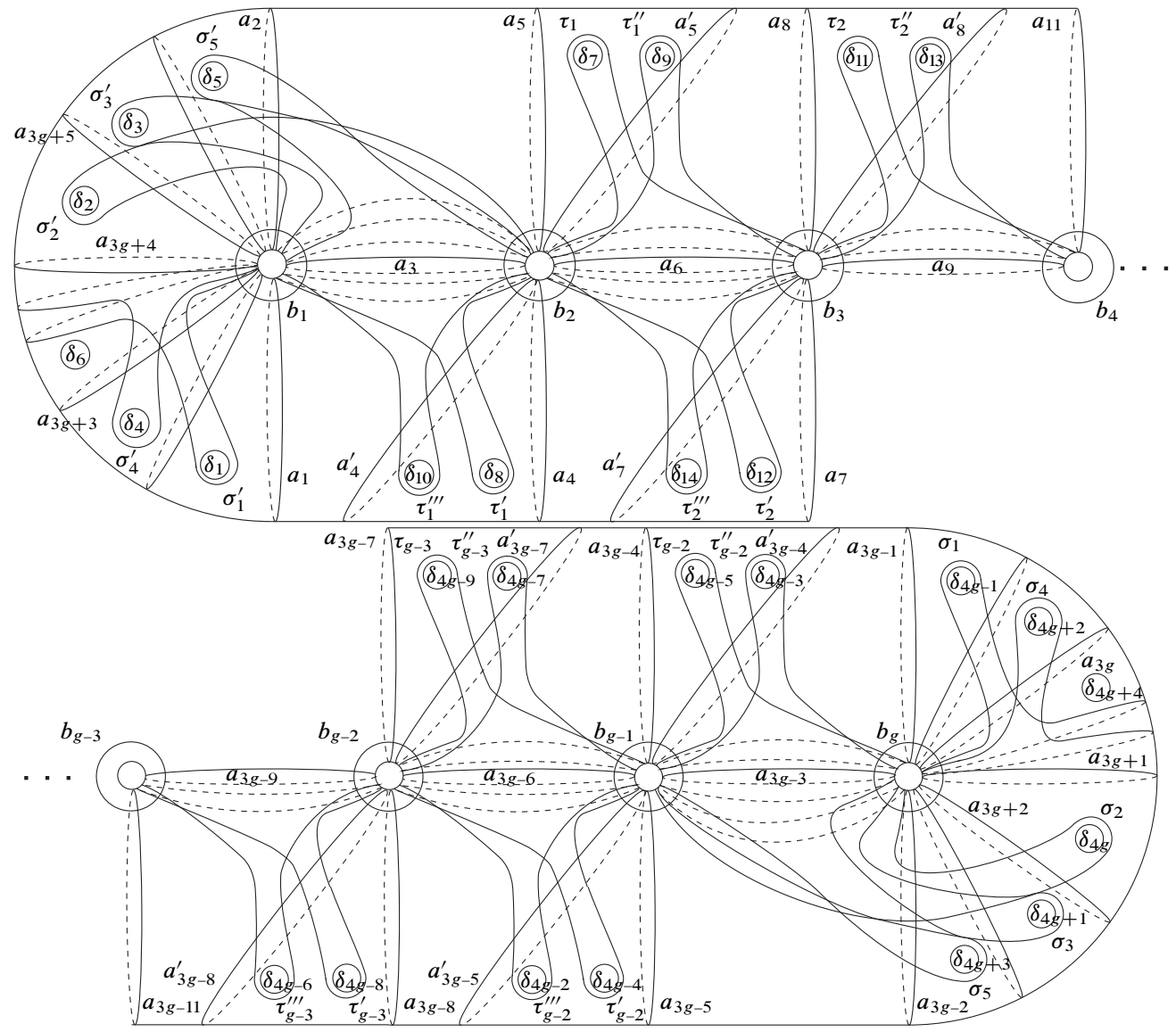

Figure 12: Relation $\left(\mathrm{H}_{g}\right)$ for $g \geq 3$

Combining these relations and applying commutativity relations and conjugations, we obtain a relation

(C2) $\quad a_{7}^{\prime} a_{8} \delta_{1} \delta_{2} \delta_{3} \delta_{4} \delta_{5} \delta_{6} \delta_{7} \delta_{8} \delta_{9} \delta_{10}$

$$
\begin{gathered}
\equiv \bar{a}_{5} \bar{a}_{4}^{\prime} a_{5} a_{4}^{\prime} \bar{a}_{3} b_{2} a_{3} a_{6} b_{2} \bar{a}_{6} \tau_{1} \tau_{1}^{\prime \prime} a_{4} a_{5}^{\prime} \bar{a}_{6} b_{2} a_{6} a_{3} b_{2} \bar{a}_{3} \tau_{1}^{\prime} \tau_{1}^{\prime \prime \prime} \bar{a}_{1} \bar{a}_{2} \\
\cdot a_{1} a_{2} \beta_{1} \sigma_{1}^{\prime} \sigma_{4}^{\prime} a_{3 g+3} \beta_{1}^{\prime} \sigma_{2}^{\prime} a_{1} \beta_{1}^{\prime \prime} \sigma_{3}^{\prime} \sigma_{5}^{\prime} \\
\equiv \bar{a}_{3} b_{2} a_{3} a_{6} b_{2} \bar{a}_{6} \tau_{1} \tau_{1}^{\prime \prime} a_{4} a_{5}^{\prime} \bar{a}_{6} b_{2} a_{6} a_{3} b_{2} \bar{a}_{3} \tau_{1}^{\prime} \tau_{1}^{\prime \prime \prime} \\
\cdot \beta_{1} \sigma_{1}^{\prime} \sigma_{4}^{\prime} a_{3 g+3} \beta_{1}^{\prime} \sigma_{2}^{\prime} a_{1} \beta_{1}^{\prime \prime} \sigma_{3}^{\prime} \sigma_{5}^{\prime} \\
\equiv a_{4} a_{5}^{\prime} \bar{a}_{6} b_{2} a_{6} a_{3} b_{2} \bar{a}_{3} \tau_{1}^{\prime} \tau_{1}^{\prime \prime \prime} \cdot \beta_{1} \sigma_{1}^{\prime} \sigma_{4}^{\prime} a_{3 g+3} \beta_{1}^{\prime} \sigma_{2}^{\prime} a_{1} \beta_{1}^{\prime \prime} \sigma_{3}^{\prime} \sigma_{5}^{\prime} \\
\cdot \bar{a}_{3} b_{2} a_{3} a_{6} b_{2} \bar{a}_{6} \tau_{1} \tau_{1}^{\prime \prime} .
\end{gathered}
$$



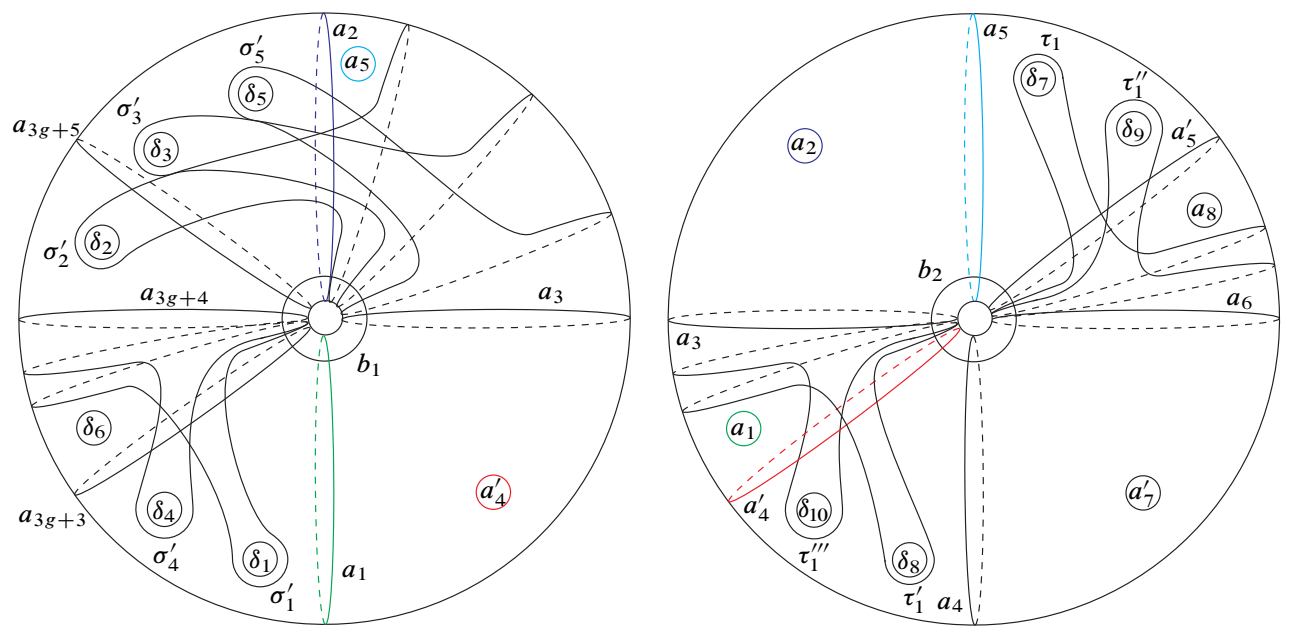

Figure 13: Relations (A) and (B)

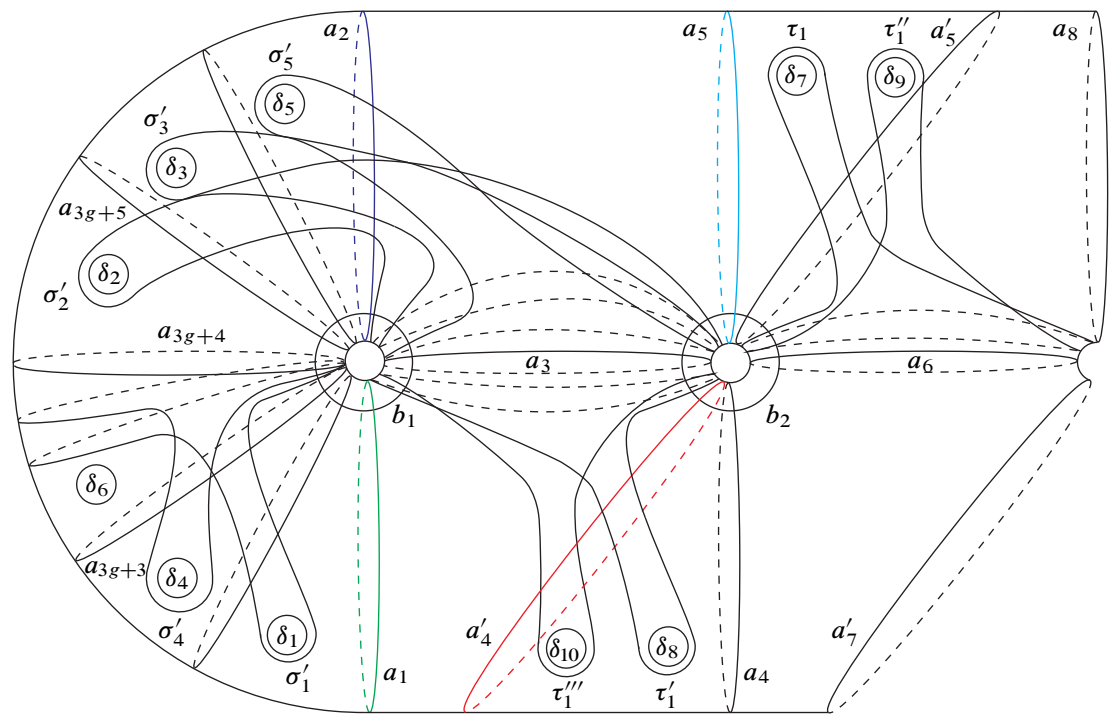

Figure 14: Embeddings of two copies of $\Sigma_{1,8}$ into $\Sigma_{2,12}$ (I)

We next consider relation (C2) and another copy of relation (B) for simple closed curves shown in Figure 15:

$$
a_{4} a_{5}^{\prime} a_{10}^{\prime} a_{11} \delta_{11} \delta_{12} \delta_{13} \delta_{14} \equiv a_{8} a_{7}^{\prime} \bar{a}_{6} b_{3} a_{6} a_{9} b_{3} \bar{a}_{9} \tau_{2} \tau_{2}^{\prime \prime} a_{7} a_{8}^{\prime} \bar{a}_{9} b_{3} a_{9} a_{6} b_{3} \bar{a}_{6} \tau_{2}^{\prime} \tau_{2}^{\prime \prime \prime}
$$

We embed $\Sigma_{2,12}$ in Figure 14 and $\Sigma_{1,8}$ in Figure 15 into $\Sigma_{3,16}$ as shown in Figure 16. 


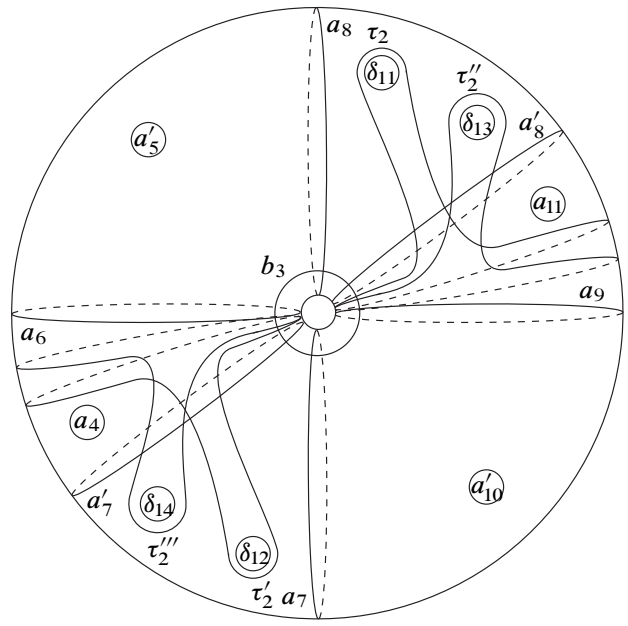

Figure 15: Another relation (B)

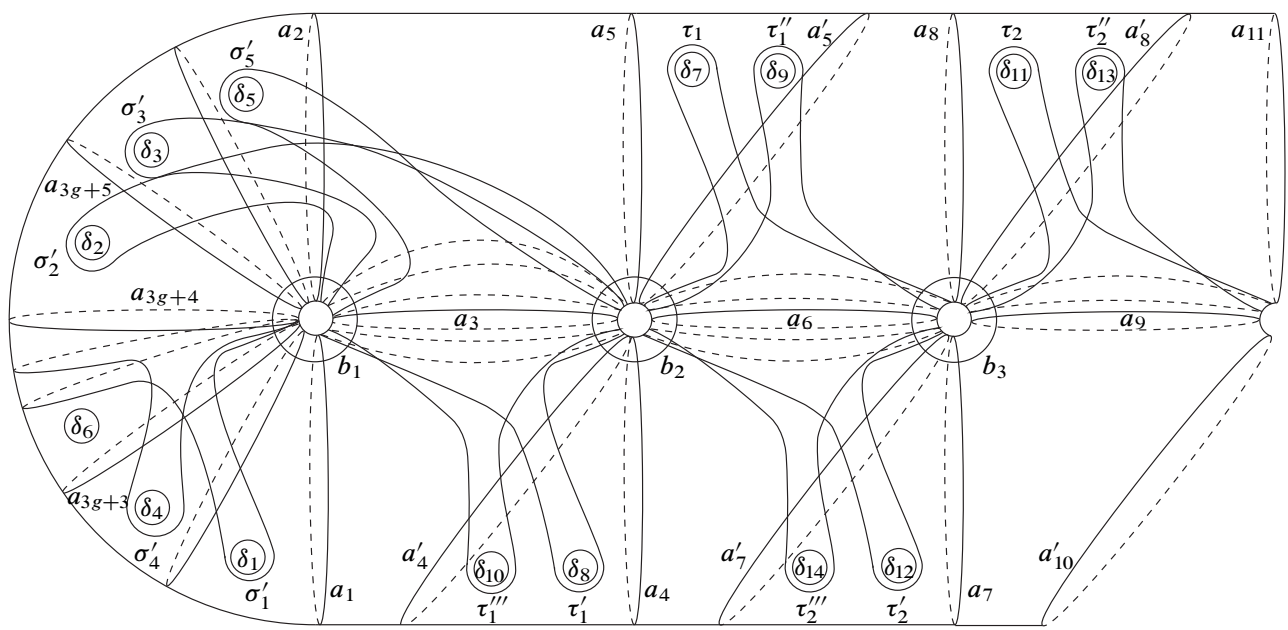

Figure 16: Embeddings of $\Sigma_{2,12}$ and $\Sigma_{1,8}$ into $\Sigma_{3,16}$

Combining these relations and applying commutativity relations and conjugations, we obtain a relation

(C3)

$$
\begin{aligned}
& a_{10}^{\prime} a_{11} \delta_{1} \delta_{2} \delta_{3} \delta_{4} \delta_{5} \delta_{6} \delta_{7} \delta_{8} \delta_{9} \delta_{10} \delta_{11} \delta_{12} \delta_{13} \delta_{14} \\
& \equiv \bar{a}_{7}^{\prime} \bar{a}_{8} a_{8} a_{7}^{\prime} \bar{a}_{6} b_{3} a_{6} a_{9} b_{3} \bar{a}_{9} \tau_{2} \tau_{2}^{\prime \prime} a_{7} a_{8}^{\prime} \bar{a}_{9} b_{3} a_{9} a_{6} b_{3} \bar{a}_{6} \tau_{2}^{\prime} \tau_{2}^{\prime \prime \prime} \bar{a}_{4} \bar{a}_{5}^{\prime} \\
& \cdot a_{4} a_{5}^{\prime} \bar{a}_{6} b_{2} a_{6} a_{3} b_{2} \bar{a}_{3} \tau_{1}^{\prime} \tau_{1}^{\prime \prime \prime} \cdot \beta_{1} \sigma_{1}^{\prime} \sigma_{4}^{\prime} a_{3 g+3} \beta_{1}^{\prime} \sigma_{2}^{\prime} a_{1} \beta_{1}^{\prime \prime} \sigma_{3}^{\prime} \sigma_{5}^{\prime} \\
& \cdot \bar{a}_{3} b_{2} a_{3} a_{6} b_{2} \bar{a}_{6} \tau_{1} \tau_{1}^{\prime \prime}
\end{aligned}
$$




$$
\begin{gathered}
\equiv \bar{a}_{6} b_{3} a_{6} a_{9} b_{3} \bar{a}_{9} \tau_{2} \tau_{2}^{\prime \prime} a_{7} a_{8}^{\prime} \bar{a}_{9} b_{3} a_{9} a_{6} b_{3} \bar{a}_{6} \tau_{2}^{\prime} \tau_{2}^{\prime \prime \prime} \cdot \bar{a}_{6} b_{2} a_{6} a_{3} b_{2} \bar{a}_{3} \tau_{1}^{\prime} \tau_{1}^{\prime \prime \prime} \\
\cdot \beta_{1} \sigma_{1}^{\prime} \sigma_{4}^{\prime} a_{3 g+3} \beta_{1}^{\prime} \sigma_{2}^{\prime} a_{1} \beta_{1}^{\prime \prime} \sigma_{3}^{\prime} \sigma_{5}^{\prime} \cdot \bar{a}_{3} b_{2} a_{3} a_{6} b_{2} \bar{a}_{6} \tau_{1} \tau_{1}^{\prime \prime} \\
\equiv a_{7} a_{8}^{\prime} \bar{a}_{9} b_{3} a_{9} a_{6} b_{3} \bar{a}_{6} \tau_{2}^{\prime} \tau_{2}^{\prime \prime \prime} \cdot \bar{a}_{6} b_{2} a_{6} a_{3} b_{2} \bar{a}_{3} \tau_{1}^{\prime} \tau_{1}^{\prime \prime \prime} \\
\cdot \beta_{1} \sigma_{1}^{\prime} \sigma_{4}^{\prime} a_{3 g+3} \beta_{1}^{\prime} \sigma_{2}^{\prime} a_{1} \beta_{1}^{\prime \prime} \sigma_{3}^{\prime} \sigma_{5}^{\prime} \cdot \bar{a}_{3} b_{2} a_{3} a_{6} b_{2} \bar{a}_{6} \tau_{1} \tau_{1}^{\prime \prime} \\
\cdot \bar{a}_{6} b_{3} a_{6} a_{9} b_{3} \bar{a}_{9} \tau_{2} \tau_{2}^{\prime \prime} .
\end{gathered}
$$

We repeat similar procedures by making use of $g-4$ copies of relation (B):

$$
\begin{aligned}
& a_{3 i-5} a_{3 i-4}^{\prime} a_{3 i+1} a_{3 i+2} \delta_{4 i-1} \delta_{4 i} \delta_{4 i+1} \delta_{4 i+2} \\
& \equiv a_{3 i-1} a_{3 i-2}^{\prime} \bar{a}_{3 i-3} b_{i} a_{3 i-3} a_{3 i} b_{i} \bar{a}_{3 i} \tau_{i-1} \tau_{i-1}^{\prime \prime} \\
& \cdot a_{3 i-2} a_{3 i-1}^{\prime} \bar{a}_{3 i} b_{i} a_{3 i} a_{3 i-3} b_{i} \bar{a}_{3 i-3} \tau_{i-1}^{\prime} \tau_{i-1}^{\prime \prime \prime}
\end{aligned}
$$

for $i=4, \ldots, g-1$ to obtain relations (C4), (C5), . a and

$(\mathrm{C}(g-1)) a_{3 g-2} a_{3 g-1} \delta_{1} \delta_{2} \cdots \delta_{4 g-3} \delta_{4 g-2}$

$$
\begin{aligned}
\equiv & a_{3 g-5} a_{3 g-4}^{\prime} \prod_{i=g-1}^{2} \bar{a}_{3 i} b_{i} a_{3 i} a_{3 i-3} b_{i} \bar{a}_{3 i-3} \tau_{i-1}^{\prime} \tau_{i-1}^{\prime \prime \prime} \\
& \cdot \beta_{1} \sigma_{1}^{\prime} \sigma_{4}^{\prime} a_{3 g+3} \beta_{1}^{\prime} \sigma_{2}^{\prime} a_{1} \beta_{1}^{\prime \prime} \sigma_{3}^{\prime} \sigma_{5}^{\prime} \prod_{i=2}^{g-1} \bar{a}_{3 i-3} b_{i} a_{3 i-3} a_{3 i} b_{i} \bar{a}_{3 i} \tau_{i-1} \tau_{i-1}^{\prime \prime}
\end{aligned}
$$

for simple closed curves on $\Sigma_{g-1,4 g}$ shown in Figure 17.

We finally consider the other copy of relation (A) for simple closed curves shown in Figure 18:

$$
\begin{aligned}
& a_{3 g-5} a_{3 g-4}^{\prime} \delta_{4 g+1} \delta_{4 g+2} \delta_{4 g+4} \delta_{4 g} \delta_{4 g+1} \delta_{4 g+3} \\
& \equiv a_{3 g-1} a_{3 g+1} b_{g} \bar{a}_{3 g+1} \sigma_{1} \sigma_{4} a_{3 g} a_{3 g-3} b_{g} \bar{a}_{3 g-3} \sigma_{2} \\
& \cdot a_{3 g-1} a_{3 g-3} a_{3 g+2} b_{g} \bar{a}_{3 g+2} \bar{a}_{3 g-3} \sigma_{3} \sigma_{5} a_{3 g-2}
\end{aligned}
$$

Applying commutativity relations and conjugations, we obtain a relation

$$
\begin{aligned}
& a_{3 g-5} a_{3 g-4}^{\prime} \delta_{4 g-1} \delta_{4 g} \delta_{4 g+1} \delta_{4 g+2} \delta_{4 g+3} \delta_{4 g+4} \\
& \equiv a_{3 g-2} a_{3 g-1} a_{3 g+1} b_{g} \bar{a}_{3 g+1} \sigma_{1} \sigma_{4} a_{3 g} a_{3 g-3} b_{g} \\
& \cdot \bar{a}_{3 g-3} \sigma_{2} a_{3 g-1} a_{3 g-3} a_{3 g+2} b_{g} \bar{a}_{3 g+2} \bar{a}_{3 g-3} \sigma_{3} \sigma_{5} \\
& \equiv a_{3 g-2} a_{3 g-1} \beta_{g} \sigma_{1} \sigma_{4} a_{3 g} \beta_{g}^{\prime} \sigma_{2} a_{3 g-1} \beta_{g}^{\prime \prime} \sigma_{3} \sigma_{5} .
\end{aligned}
$$

Note that

$$
\beta_{g} \equiv a_{3 g+1} b_{g} \bar{a}_{3 g+1}, \quad \beta_{g}^{\prime} \equiv a_{3 g-3} b_{g} \bar{a}_{3 g-3}, \quad \beta_{g}^{\prime \prime} \equiv a_{3 g-3} a_{3 g+2} b_{g} \bar{a}_{3 g+2} \bar{a}_{3 g-3}
$$




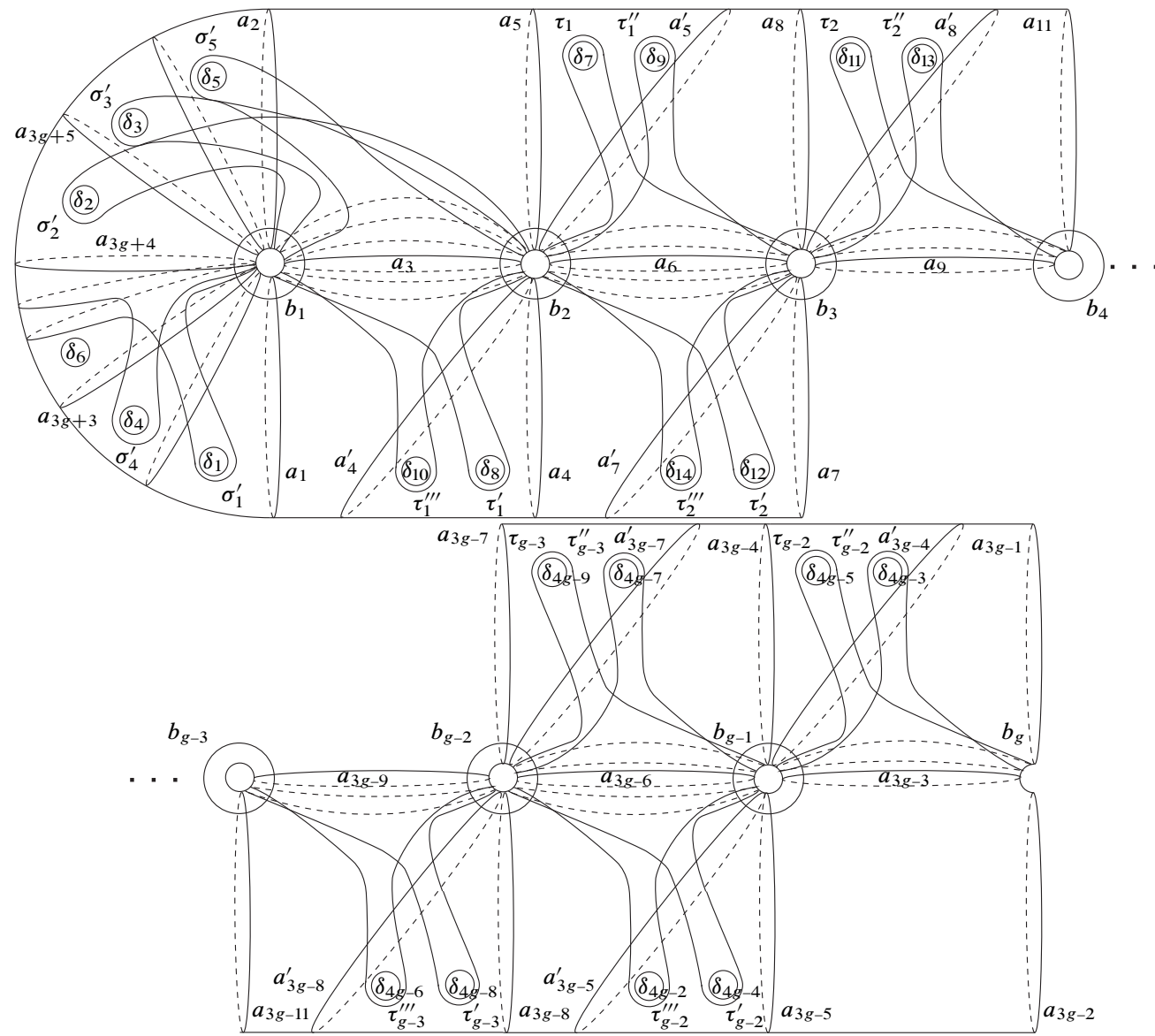

Figure 17: Relation $(\mathrm{C}(g-1))$

by Remark 2.2. We embed $\Sigma_{g-1,4 g}$ in Figure 17 and $\Sigma_{1,8}$ in Figure 18 into $\Sigma_{g, 4 g+4}$ as shown in Figure 12.

Combining these relations and applying commutativity relations and conjugations, we obtain relation $\left(\mathrm{H}_{g}\right)$. Note that $\beta_{i} \equiv a_{3 i-3} b_{i} \bar{a}_{3 i-3}, \beta_{i}^{\prime} \equiv a_{3 i} b_{i} \bar{a}_{3 i}, \beta_{i}^{\prime \prime} \equiv \bar{a}_{3 i-3} b_{i} a_{3 i-3}$ and $\beta_{i}^{\prime \prime \prime} \equiv \bar{a}_{3 i} b_{i} a_{3 i}(i=2, \ldots, g-1)$ by Remark 2.2. Thus we complete the proof of Theorem 3.1.

\subsection{Genus two}

In this subsection we construct a relation on $\Sigma_{2,12}$ similar to relations constructed in the previous subsection. 


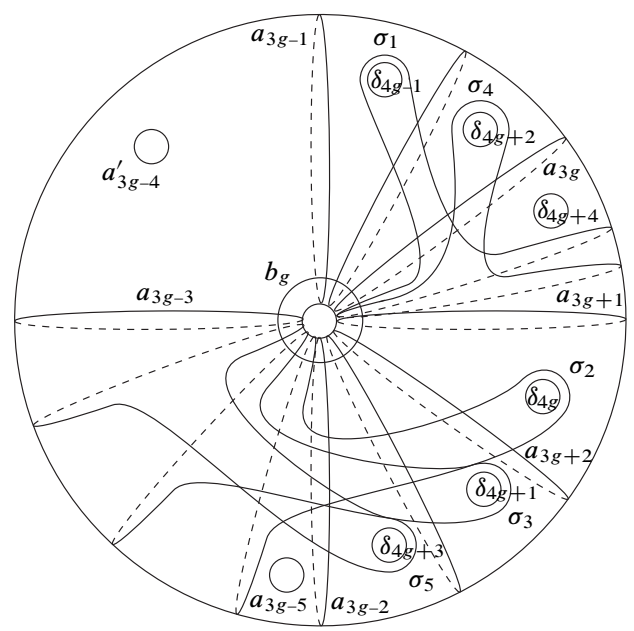

Figure 18: The other relation (A)

Theorem 3.2 Relation $\left(\mathrm{H}_{2}\right)$ For simple closed curves in the interior of $\Sigma_{2,12}$ shown in Figure 19, we have the relation $\delta_{1} \delta_{2} \delta_{3} \delta_{4} \delta_{5} \delta_{6} \delta_{7} \delta_{8} \delta_{9} \delta_{10} \delta_{11} \delta_{12} \equiv \beta_{1} \sigma_{1}^{\prime} \sigma_{4}^{\prime} a_{13} \beta_{1}^{\prime} \sigma_{2}^{\prime} a_{1} \beta_{1}^{\prime \prime} \sigma_{3}^{\prime} \sigma_{5}^{\prime} \beta_{2} \sigma_{1} \sigma_{4} a_{12} \beta_{2}^{\prime} \sigma_{2} a_{5} \beta_{2}^{\prime \prime} \sigma_{3} \sigma_{5}$ in $\mathcal{M}_{2,12}$, where

$$
\begin{array}{ll}
\beta_{1}:=a_{2}\left(b_{1}\right), & \beta_{1}^{\prime}:=a_{3}\left(b_{1}\right), \quad \beta_{1}^{\prime \prime}:=a_{3} a_{9}\left(b_{1}\right), \\
\beta_{2}:=a_{4}\left(b_{2}\right), & \beta_{2}^{\prime}:=a_{3}\left(b_{2}\right), \quad \beta_{2}^{\prime \prime}:=a_{3} a_{8}\left(b_{2}\right) .
\end{array}
$$

Proof We first consider two copies of relation (A) for simple closed curves shown in Figure 20:

$$
\begin{aligned}
a_{5} a_{14} \delta_{7} \delta_{8} \delta_{9} \delta_{10} \delta_{11} \delta_{12} & \equiv a_{1} a_{2} b_{1} \bar{a}_{2} \sigma_{1}^{\prime} \sigma_{4}^{\prime} a_{13} a_{3} b_{1} \bar{a}_{3} \sigma_{2}^{\prime} a_{1} a_{3} a_{9} b_{1} \bar{a}_{9} \bar{a}_{3} \sigma_{3}^{\prime} \sigma_{5}^{\prime} a_{15} \\
a_{1} a_{15} \delta_{1} \delta_{2} \delta_{3} \delta_{4} \delta_{5} \delta_{6} & \equiv a_{5} a_{4} b_{2} \bar{a}_{4} \sigma_{1} \sigma_{4} a_{12} a_{3} b_{2} \bar{a}_{3} \sigma_{2} a_{5} a_{3} a_{8} b_{2} \bar{a}_{8} \bar{a}_{3} \sigma_{3} \sigma_{5} a_{14} .
\end{aligned}
$$

Applying commutativity relations and conjugations, we obtain relations

$$
\begin{aligned}
a_{5} a_{14} \delta_{7} \delta_{8} \delta_{9} \delta_{10} \delta_{11} \delta_{12} & \equiv a_{15} a_{1} \beta_{1} \sigma_{1}^{\prime} \sigma_{4}^{\prime} a_{13} \beta_{1}^{\prime} \sigma_{2}^{\prime} a_{1} \beta_{1}^{\prime \prime} \sigma_{3}^{\prime} \sigma_{5}^{\prime} \\
a_{1} a_{15} \delta_{1} \delta_{2} \delta_{3} \delta_{4} \delta_{5} \delta_{6} & \equiv a_{14} a_{5} \beta_{2} \sigma_{1} \sigma_{4} a_{12} \beta_{2}^{\prime} \sigma_{2} a_{5} \beta_{2}^{\prime \prime} \sigma_{3} \sigma_{5} .
\end{aligned}
$$

Note that $\beta_{1} \equiv a_{2} b_{1} \bar{a}_{2}, \beta_{1}^{\prime} \equiv a_{3} b_{1} \bar{a}_{3}, \beta_{1}^{\prime \prime} \equiv a_{3} a_{9} b_{1} \bar{a}_{9} \bar{a}_{3}, \beta_{2} \equiv a_{4} b_{2} \bar{a}_{4}, \beta_{2}^{\prime} \equiv a_{3} b_{2} \bar{a}_{3}$ and $\beta_{2}^{\prime \prime} \equiv a_{3} a_{8} b_{2} \bar{a}_{8} \bar{a}_{3}$ by Remark 2.2.

Combining these relations and applying commutativity relations and conjugations, we obtain relation $\left(\mathrm{H}_{2}\right)$. Thus we complete the proof of Theorem 3.2. 


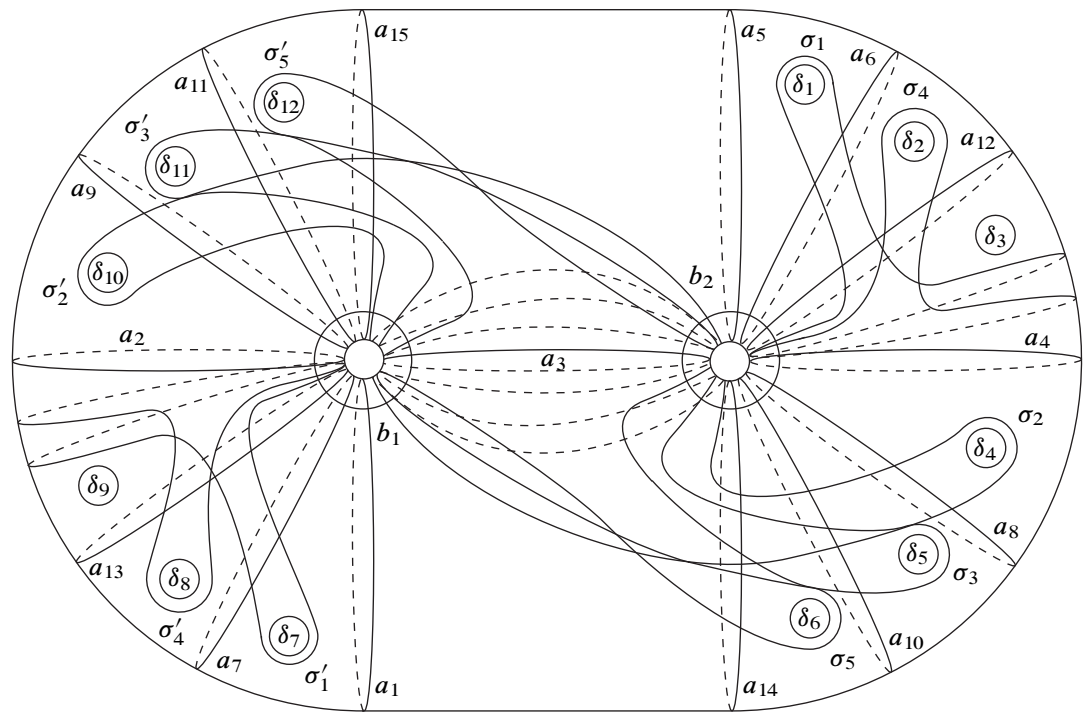

Figure 19: Embeddings of two copies of $\Sigma_{1,8}$ into $\Sigma_{2,12}$ (II)
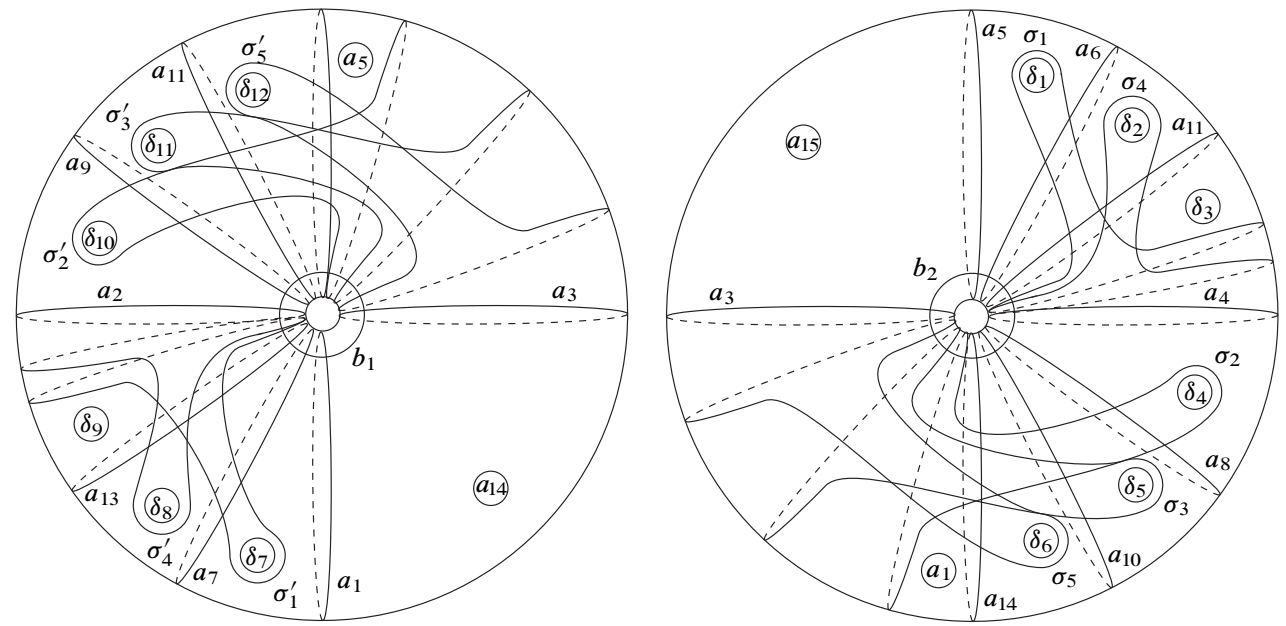

Figure 20: Two copies of relation (A)

\section{Sections of Lefschetz fibrations}

In this section we show that the relation constructed in the previous section gives an explicit topological description of $4 g+4$ disjoint $(-1)$-sections of a hyperelliptic Lefschetz fibration of genus $g$ on the manifold $\mathbb{C P}^{2} \#(4 g+5) \overline{\mathbb{C P}}^{2}$. 
We begin with a definition of Lefschetz fibrations (see [4; 8]).

Definition 4.1 Let $M$ be a closed oriented smooth 4-manifold. A smooth map $f: M \rightarrow S^{2}$ is called a Lefschetz fibration of genus $g$ if it satisfies the following conditions:

(i) $f$ has finitely many critical values $b_{1}, \ldots, b_{n} \in S^{2}$ and $f$ is a smooth fiber bundle over $S^{2}-\left\{b_{1}, \ldots, b_{n}\right\}$ with fiber $\Sigma_{g, 0}$

(ii) for each $i(i=1, \ldots, n)$, there exists a unique critical point $p_{i}$ in the singular fiber $f^{-1}\left(b_{i}\right)$ such that $f$ is locally written as $f\left(z_{1}, z_{2}\right)=z_{1}^{2}+z_{2}^{2}$ with respect to some local complex coordinates around $p_{i}$ and $b_{i}$ which are compatible with orientations of $M$ and $S^{2}$

(iii) no fiber contains a $(-1)-$ sphere.

Remark 4.2 We always assume that a Lefschetz fibration is relatively minimal, it has at most one critical point on each fiber, and the genus of the base is equal to zero. A more general definition can be found in [4, Chapter 8].

Suppose that $g \geq 2$. According to theorems of Kas and Matsumoto, there exists a one-to-one correspondence between the isomorphism classes of Lefschetz fibrations and the equivalence classes of positive relators modulo simultaneous conjugations

$$
c_{1} \cdots c_{n} \sim{ }_{W}\left(c_{1}\right) \cdots_{W}\left(c_{n}\right),
$$

and elementary transformations

$$
\begin{aligned}
& c_{1} \cdots c_{i} \cdot c_{i+1} \cdots c_{n} \sim c_{1} \cdots c_{i+1} \cdot{ }_{c_{i+1}^{-1}}\left(c_{i}\right) \cdots c_{n}, \\
& c_{1} \cdots c_{i} \cdot c_{i+1} \cdots c_{n} \sim c_{1} \cdots c_{i}\left(c_{i+1}\right) \cdot c_{i} \cdots c_{n},
\end{aligned}
$$

where $c_{1} \cdots c_{n} \in \mathcal{R}_{g, 0}$ is a positive relator in the generators $\mathcal{S}_{g, 0}$ and $W \in \mathcal{F}_{g, 0}$. This correspondence is described by using the holonomy (or monodromy) homomorphism induced by the classifying map of $f$ restricted on $S^{2}-\left\{b_{1}, \ldots, b_{n}\right\}$ (see $[4 ; 8]$ ).

Definition 4.3 Let $f: M \rightarrow S^{2}$ be a Lefschetz fibration of genus $g$. A smooth map $s: S^{2} \rightarrow M$ is called a section of $f$ if it satisfies $f \circ s=\mathrm{id}_{S^{2}}$. A section $s$ of $f$ is an embedding of $S^{2}$ into $M$. The self-intersection number of the homology class $s_{*}\left(\left[S^{2}\right]\right) \in H_{2}(M ; \mathbb{Z})$ is called the self-intersection number of $s$. A section of $f$ with self-intersection number $k$ is often called a $k$-section. 
For a positive integer $r$, we attach $r$ disks to the boundary components of $\Sigma_{g, r}$ to obtain a closed surface $\Sigma_{g, 0}$ and an embedding $\Sigma_{g, r} \hookrightarrow \Sigma_{g, 0}$. This embedding induces a natural commutative diagram

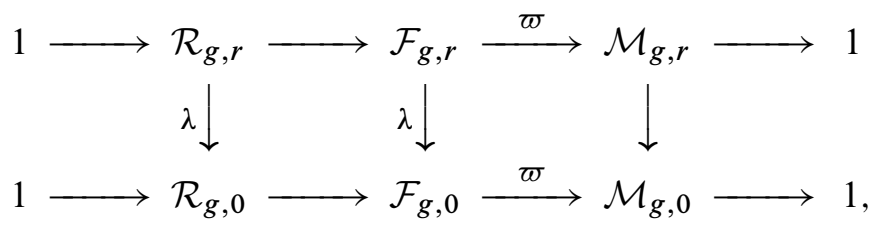

where the two horizontal sequences are exact. If two words $W_{1}$ and $W_{2}$ in $\mathcal{F}_{g, r}$ satisfy $W_{1} \equiv W_{2}$, then we have $\lambda\left(W_{1}\right) \equiv \lambda\left(W_{2}\right)$ in $\mathcal{F}_{g, 0}$. In this case we call the relation $W_{1} \equiv W_{2}$ a lift of the relation $\lambda\left(W_{1}\right) \equiv \lambda\left(W_{2}\right)$.

Lemma 4.4 $[1 ; 2 ; 11]$ Let $f: M \rightarrow S^{2}$ be a Lefschetz fibration of genus $g$ and $c_{1} \cdots c_{n} \in \mathcal{R}_{g, 0}$ a positive relator corresponding to $f$. Suppose that there exists a relation $a_{1} \cdots a_{n} \equiv \delta_{1}^{k_{1}} \cdots \delta_{r}^{k_{r}}\left(a_{1}, \ldots, a_{n} \in \mathcal{S}_{g, r}, k_{1}, \ldots, k_{r}>0\right)$ in $\mathcal{F}_{g, r}$ which is a lift of the relation $c_{1} \cdots c_{n} \equiv 1$ in $\mathcal{F}_{g, 0}$, where $\delta_{1}, \ldots, \delta_{r}$ are simple closed curves parallel to the boundary components of $\Sigma_{g, r}$. Then $f$ admits disjoint $r$ sections $s_{1}, \ldots, s_{r}: S^{2} \rightarrow M$ with self-intersection number $-k_{1}, \ldots,-k_{r}$, respectively.

For a chain $\left(c_{1}, \ldots, c_{2 g+1}\right)$ of length $2 g+1$ on $\Sigma_{g, 0}$, we obtain a Lefschetz fibration $X_{g} \rightarrow S^{2}$ of genus $g$ associated to the hyperelliptic relation

$$
\left(c_{1} \cdots c_{2 g+1} c_{2 g+1} \cdots c_{1}\right)^{2} \equiv 1 \quad \text { in } \mathcal{F}_{g, 0} .
$$

The total space $X_{g}$ of this fibration is known to be diffeomorphic to $\mathbb{C P}^{2} \#(4 g+5) \overline{\mathbb{C P}}^{2}$ (see $[4 ; 5])$.

We denote the positive word on the right-hand side of relation $\left(\mathrm{H}_{g}\right)$ by $U_{g}$ for $g \geq 2$. We consider the above embedding $\Sigma_{g, r} \hookrightarrow \Sigma_{g, 0}$ and the commutative diagram for $r=4 g+4$. By Theorems 3.1 and 3.2, relation $\left(\mathrm{H}_{g}\right): U_{g} \equiv \delta_{1} \delta_{2} \cdots \delta_{4 g+3} \delta_{4 g+4}$ in $\mathcal{F}_{g, 4 g+4}$ is a lift of the relation $\lambda\left(U_{g}\right) \equiv 1$ in $\mathcal{F}_{g, 0}$. This implies that the Lefschetz fibration $Y_{g} \rightarrow S^{2}$ of genus $g$ associated to the relation $\lambda\left(U_{g}\right) \equiv 1$ admits disjoint $4 g+4$ sections with self-intersection number -1 by virtue of Lemma 4.4.

Theorem 4.5 The two Lefschetz fibrations $X_{g}$ and $Y_{g}$ are isomorphic to each other.

Proof Suppose that $g \geq 3$. We set

$$
\begin{aligned}
& c_{1}:=\lambda\left(a_{1}\right), \quad c_{2 i}:=\lambda\left(b_{i}\right) \quad(i=1, \ldots, g), \\
& c_{2 g+1}:=\lambda\left(a_{3 g-1}\right), \quad c_{2 i+1}:=\lambda\left(a_{3 i}\right) \quad(i=1, \ldots, g-1) .
\end{aligned}
$$


Since $\left(a_{1}, b_{1}, a_{3}, b_{2}, \ldots, a_{3 g-3}, b_{g}, a_{3 g-1}\right)$ is a chain of length $2 g+1$ on $\Sigma_{g, 4 g+4}$, $\left(c_{1}, c_{2}, c_{3}, c_{4}, \ldots, c_{2 g-1}, c_{2 g}, c_{2 g+1}\right)$ is a chain of length $2 g+1$ on $\Sigma_{g, 0}$. It is easily seen from Figure 12 that

$$
\begin{gathered}
\lambda\left(a_{3 g+3}\right)=\lambda\left(a_{3 g+4}\right)=\lambda\left(a_{3 g+5}\right)=\lambda\left(\sigma_{1}^{\prime}\right)=\lambda\left(\sigma_{4}^{\prime}\right)=c_{1}, \\
\lambda\left(a_{3 g}\right)=\lambda\left(a_{3 g+1}\right)=\lambda\left(a_{3 g+2}\right)=\lambda\left(\sigma_{1}\right)=\lambda\left(\sigma_{4}\right)=c_{2 g+1}, \\
\lambda\left(\sigma_{2}^{\prime}\right)=\lambda\left(\sigma_{3}^{\prime}\right)=\lambda\left(\sigma_{5}^{\prime}\right)=c_{3}, \\
\lambda\left(\sigma_{2}\right)=\lambda\left(\sigma_{3}\right)=\lambda\left(\sigma_{5}\right)=c_{2 g-1}, \\
\lambda\left(\tau_{i-1}^{\prime}\right)=\lambda\left(\tau_{i-1}^{\prime \prime \prime}\right)=c_{2 i-1}, \\
\lambda\left(\tau_{i-1}\right)=\lambda\left(\tau_{i-1}^{\prime \prime}\right)=c_{2 i+1},
\end{gathered}
$$

for $i=2, \ldots, g-1$. Hence we obtain

$$
\begin{array}{r}
\lambda\left(U_{g}\right)=\prod_{i=g-1}^{2}\left(\bar{c}_{2 i+1}\left(c_{2 i}\right)_{c_{2 i-1}}\left(c_{2 i}\right) \cdot c_{2 i-1}^{2}\right) \cdot c_{1}\left(c_{2}\right) \cdot c_{1}^{3} \cdot c_{3}\left(c_{2}\right) \cdot c_{3} c_{1} \cdot c_{1} c_{3}\left(c_{2}\right) \cdot c_{3}^{2} \\
\cdot \prod_{i=2}^{g-1}\left(\bar{c}_{2 i-1}\left(c_{2 i}\right)_{c_{2 i+1}}\left(c_{2 i}\right) \cdot c_{2 i+1}^{2}\right) \cdot c_{2 g+1}\left(c_{2 g}\right) \cdot c_{2 g+1}^{3} \cdot c_{2 g-1}\left(c_{2 g}\right) \\
\cdot c_{2 g-1} c_{2 g+1} \cdot c_{2 g-1} c_{2 g+1}\left(c_{2 g}\right) \cdot c_{2 g-1}^{2}
\end{array}
$$

We now prove that $\lambda\left(U_{g}\right) \sim\left(c_{1} \cdots c_{2 g+1} c_{2 g+1} \cdots c_{1}\right)^{2}$ for $g \geq 3$. Applying elementary transformations (including cyclic permutations), we obtain the following sequence of equivalences:

$$
\begin{gathered}
\lambda\left(U_{g}\right) \sim c_{2 g-1} \cdot \prod_{i=g-1}^{2}\left(\bar{c}_{2 i+1}\left(c_{2 i}\right) \cdot c_{2 i-1} c_{2 i} c_{2 i-1}\right) \cdot c_{1} c_{2} c_{1}^{2} c_{3} c_{2} c_{1} c_{3} \cdot c_{1}\left(c_{2}\right) \cdot c_{3} \\
\cdot \prod_{i=2}^{g-1}\left(\bar{c}_{2 i-1}\left(c_{2 i}\right) \cdot c_{2 i+1} c_{2 i} c_{2 i+1}\right) \\
\cdot c_{2 g+1} c_{2 g} c_{2 g+1}^{2} c_{2 g-1} c_{2 g} c_{2 g+1} c_{2 g-1} \cdot c_{2 g+1}\left(c_{2 g}\right) \\
\sim \prod_{i=g-1}^{2}\left(c_{2 i+1} \cdot \bar{c}_{2 i+1}\left(c_{2 i}\right) \cdot c_{2 i-1} c_{2 i}\right) \cdot c_{3} c_{1} c_{2} c_{1}^{2} c_{3} c_{2} c_{1} c_{3} \cdot c_{1}\left(c_{2}\right) \\
\cdot \prod_{i=2}^{g-1}\left(c_{2 i-1} \cdot \bar{c}_{2 i-1}\left(c_{2 i}\right) \cdot c_{2 i+1} c_{2 i}\right) \\
\sim c_{1} c_{2 g+1} \cdot \prod_{i=g-1}^{2} c_{2 i} c_{2 i+1} c_{2 i-1} c_{2 i} \cdot c_{3} c_{2} c_{1}^{2} c_{3} c_{2} c_{1} c_{3} \cdot c_{1}\left(c_{2}\right)
\end{gathered}
$$




$$
\begin{aligned}
& \cdot \prod_{i=2}^{g-1} c_{2 i} c_{2 i-1} c_{2 i+1} c_{2 i} \cdot c_{2 g-1} c_{2 g} c_{2 g+1}^{2} c_{2 g-1} c_{2 g} c_{2 g+1} c_{2 g-1} \cdot c_{2 g+1}\left(c_{2 g}\right) \\
& \sim \prod^{3} c_{i} c_{i+1} \cdot c_{3} c_{2} c_{1}^{2} c_{3} c_{2} c_{1} c_{3} \cdot c_{1}\left(c_{2}\right) \cdot c_{1} \\
& i=2 g-2 \\
& \cdot \prod_{i=3}^{2 g-2} c_{i+1} c_{i} \cdot c_{2 g-1} c_{2 g} c_{2 g+1}^{2} c_{2 g-1} c_{2 g} c_{2 g+1} c_{2 g-1} \cdot c_{2 g+1}\left(c_{2 g}\right) \cdot c_{2 g+1} \\
& \sim \prod_{i=2}^{3} c_{i} c_{i+1} \cdot c_{3} c_{2} c_{3} c_{1}^{2} c_{2} c_{1}^{2} c_{3} c_{2} \\
& \begin{array}{r}
i=2 g-2 \quad \cdot \prod_{i=3}^{2 g-2} c_{i+1} c_{i} \cdot c_{2 g-1} c_{2 g} c_{2 g} \\
\sim \prod_{i=2 g-2}^{3} c_{i} c_{i+1} \cdot c_{2} c_{3} c_{2} c_{1} c_{2} c_{1} c_{2} c_{1} c_{3} c_{2}
\end{array} \\
& i=2 g-2 \quad 2 g-2 \\
& \cdot \prod_{i=3}^{2 g} c_{i+1} c_{i} \cdot c_{2 g} c_{2 g-1} c_{2 g} c_{2 g+1} c_{2 g} c_{2 g+1} c_{2 g} c_{2 g+1} c_{2 g-1} c_{2 g} \\
& \sim \prod_{i=2 g-2}^{2} c_{i} c_{i+1} \cdot c_{1} c_{2} c_{1} c_{1} c_{2} c_{1} \\
& \cdot \prod_{i=2}^{2 g-1} c_{i+1} c_{i} \cdot c_{2 g+1} c_{2 g} c_{2 g+1} c_{2 g+1} c_{2 g} c_{2 g+1} c_{2 g-1} c_{2 g} \\
& \sim c_{2 g} c_{2 g+1} c_{2 g-1} c_{2 g} \cdot \prod_{i=2 g-2}^{1} c_{i} c_{i+1} \cdot c_{1} c_{1} \cdot \prod_{i=1}^{2 g} c_{i+1} c_{i} \cdot c_{2 g+1} c_{2 g+1} \\
& \sim \prod_{i=2 g}^{1} c_{i} c_{i+1} \cdot c_{1} c_{1} \cdot \prod_{i=1}^{2 g} c_{i+1} c_{i} \cdot c_{2 g+1} c_{2 g+1} \\
& \sim \prod_{i=2 g}^{1} c_{i} \cdot \prod_{i=2 g+1}^{2} c_{i} \cdot c_{1} c_{1} \cdot \prod_{i=2}^{2 g+1} c_{i} \cdot \prod_{i=1}^{2 g} c_{i} \cdot c_{2 g+1} c_{2 g+1} \\
& =\prod_{i=2 g}^{1} c_{i} \cdot \prod_{i=2 g+1}^{1} c_{i} \cdot \prod_{i=1}^{2 g+1} c_{i} \cdot \prod_{i=1}^{2 g+1} c_{i} \cdot c_{2 g+1} \\
& \sim \prod_{i=2}^{1} c_{i} \cdot \prod_{i=2 g+1}^{1} c_{i} \cdot \prod_{i=1}^{2 g+1} c_{i} \cdot \prod_{i=1}^{2 g+1} c_{i} \\
& \sim \prod_{i=1}^{2 g+1} c_{i} \cdot \prod_{i=2 g+1}^{1} c_{i} \cdot \prod_{i=1}^{2 g+1} c_{i} \cdot \prod_{i=2 g+1}^{1} c_{i}=\left(c_{1} \cdots c_{2 g+1} c_{2 g+1} \cdots c_{1}\right)^{2} .
\end{aligned}
$$


Suppose that $g=2$. We set

$$
c_{1}:=\lambda\left(a_{1}\right), \quad c_{2}:=\lambda\left(b_{1}\right), \quad c_{3}:=\lambda\left(a_{3}\right), \quad c_{4}:=\lambda\left(b_{2}\right), \quad c_{5}:=\lambda\left(a_{5}\right) .
$$

Since $\left(a_{1}, b_{1}, a_{3}, b_{2}, a_{5}\right)$ is a chain of length 5 on $\Sigma_{2,12},\left(c_{1}, c_{2}, c_{3}, c_{4}, c_{5}\right)$ is a chain of length 5 on $\Sigma_{2,0}$. It is easily seen from Figure 19 that

$$
\begin{gathered}
\lambda\left(a_{2}\right)=\lambda\left(a_{9}\right)=\lambda\left(a_{13}\right)=\lambda\left(\sigma_{1}^{\prime}\right)=\lambda\left(\sigma_{4}^{\prime}\right)=c_{1}, \\
\lambda\left(a_{4}\right)=\lambda\left(a_{8}\right)=\lambda\left(a_{12}\right)=\lambda\left(\sigma_{1}\right)=\lambda\left(\sigma_{4}\right)=c_{5}, \\
\lambda\left(\sigma_{2}^{\prime}\right)=\lambda\left(\sigma_{3}^{\prime}\right)=\lambda\left(\sigma_{5}^{\prime}\right)=\lambda\left(\sigma_{2}\right)=\lambda\left(\sigma_{3}\right)=\lambda\left(\sigma_{5}\right)=c_{3} .
\end{gathered}
$$

Hence we obtain

$$
\lambda\left(U_{2}\right)={ }_{c_{1}}\left(c_{2}\right) \cdot c_{1}^{3} \cdot c_{3}\left(c_{2}\right) \cdot c_{3} c_{1} \cdot c_{3} c_{1}\left(c_{2}\right) \cdot c_{3}^{2} \cdot c_{5}\left(c_{4}\right) \cdot c_{5}^{3} \cdot c_{3}\left(c_{4}\right) \cdot c_{3} c_{5} \cdot c_{3} c_{5}\left(c_{4}\right) \cdot c_{3}^{2} .
$$

We now prove that $\lambda\left(U_{2}\right) \sim\left(c_{1} c_{2} c_{3} c_{4} c_{5} c_{5} c_{4} c_{3} c_{2} c_{1}\right)^{2}$. Applying elementary transformations (including cyclic permutations), we obtain the following sequence of equivalences:

$$
\begin{aligned}
\lambda\left(U_{2}\right) & \sim c_{1} c_{2} c_{1}^{2} c_{3} c_{2} c_{1} c_{3} \cdot c_{1}\left(c_{2}\right) \cdot c_{3} \cdot c_{5} c_{4} c_{5}^{2} c_{3} c_{4} c_{5} c_{3} \cdot c_{5}\left(c_{4}\right) \cdot c_{3} \\
& \sim c_{5} c_{2} c_{1}^{2} c_{3} c_{2} c_{1} c_{3} \cdot c_{1}\left(c_{2}\right) \cdot c_{3} \cdot c_{4} c_{5}^{2} c_{3} c_{4} c_{5} c_{3} \cdot c_{5}\left(c_{4}\right) \cdot c_{3} c_{1} \\
& \sim c_{2} c_{1}^{2} c_{3} c_{2} c_{1} c_{3} \cdot c_{1}\left(c_{2}\right) \cdot c_{1} c_{3} \cdot c_{4} c_{5}^{2} c_{3} c_{4} c_{5} c_{3} \cdot c_{5}\left(c_{4}\right) \cdot c_{3} c_{5} \\
& \sim c_{2} c_{1}^{2} c_{3} c_{2} c_{1} c_{3} c_{1} c_{2} c_{3} \cdot c_{4} c_{5}^{2} c_{3} c_{4} c_{5} c_{3} \cdot c_{5}\left(c_{4}\right) \cdot c_{5} c_{3} \\
& \sim c_{2} c_{1}^{2} c_{3} c_{2} c_{1} c_{3} c_{1} c_{2} c_{3} c_{4} c_{5}^{2} c_{3} c_{4} c_{5} c_{3} c_{5} c_{4} c_{3} \\
& \sim c_{1} c_{3} c_{2} c_{3} c_{1} c_{1} c_{2} c_{3} c_{4} c_{3} c_{5}^{2} c_{4} c_{5} c_{3} c_{5} c_{4} c_{3} c_{2} c_{1} \\
& \sim c_{1} c_{2} c_{3} c_{2} c_{1} c_{1} c_{2} c_{4} c_{3} c_{4} c_{5}^{2} c_{4} c_{5} c_{3} c_{5} c_{4} c_{3} c_{2} c_{1} \\
& \sim c_{1} c_{2} c_{3} c_{4} c_{2} c_{1} c_{1} c_{2} c_{3} c_{4} c_{5} c_{4} c_{5} c_{4} c_{3} c_{5} c_{4} c_{3} c_{2} c_{1} \\
& \sim c_{1} c_{2} c_{3} c_{4} c_{2} c_{1} c_{1} c_{2} c_{3} c_{5} c_{4} c_{5} c_{5} c_{4} c_{3} c_{5} c_{4} c_{3} c_{2} c_{1} \\
& \sim c_{1} c_{2} c_{3} c_{4} c_{5} c_{2} c_{1} c_{1} c_{2} c_{3} c_{4} c_{5} c_{5} c_{4} c_{3} c_{5} c_{4} c_{3} c_{2} c_{1} \\
& \sim c_{1} c_{2} c_{3} c_{4} c_{5} c_{5} c_{4} c_{3} c_{5} c_{4} c_{3} c_{2} c_{1} c_{1} c_{2} c_{3} c_{4} c_{5} c_{2} c_{1} \\
& \sim c_{1} c_{2} c_{3} c_{4} c_{5} c_{5} c_{4} c_{3} c_{2} c_{1} c_{1} c_{2} c_{3} c_{4} c_{5} c_{5} c_{4} c_{3} c_{2} c_{1} \\
& =\left(c_{1} c_{2} c_{3} c_{4} c_{5} c_{5} c_{4} c_{3} c_{2} c_{1}\right)^{2} .
\end{aligned}
$$

This completes the proof of Theorem 4.5.

The next corollary immediately follows from the theorem.

Corollary 4.6 The Lefschetz fibration $X_{g} \rightarrow S^{2}$ of genus $g$ associated to the hyperelliptic relation admits disjoint $4 g+4$ sections with self-intersection number -1 . 
By virtue of Theorem 3.2, we can even depict disjoint twelve sections of the Lefschetz fibration $Y_{2} \rightarrow S^{2}$ in a Kirby diagram of $Y_{2}-v F$, where $v F$ is an open fibered neighborhood of a regular fiber of $Y_{2}$ (see [7, Section 4]). We first construct a handle decomposition of $\Sigma_{2,0} \times D^{2}$ with one 0 -handle, four 1-handles, and one 2-handle with framing 0 from a fixed handle decomposition of $\Sigma_{2,0}$. We then attach twenty 2-handles to $\Sigma_{2,0} \times D^{2}$ along the simple closed curves $\beta_{1}, \sigma_{1}^{\prime}, \sigma_{4}^{\prime}, a_{13}, \beta_{1}^{\prime}, \sigma_{2}^{\prime}, a_{1}$, $\beta_{1}^{\prime \prime}, \sigma_{3}^{\prime}, \sigma_{5}^{\prime}, \beta_{2}, \sigma_{1}, \sigma_{4}, a_{12}, \beta_{2}^{\prime}, \sigma_{2}, a_{5}, \beta_{2}^{\prime \prime}, \sigma_{3}, \sigma_{5}$ (see Figure 19) on different fibers of $\Sigma_{2,0} \times S^{1} \rightarrow S^{1}$ with framing one less than the product framing of $\Sigma_{2,0} \times S^{1}$ to obtain a handle decomposition of $Y_{2}-v F$. Thus we have a Kirby diagram of $Y_{2}-v F$ shown in Figure 21. The framing coefficient of every component of the link but one with framing 0 is equal to -1 . Twelve disjoint sections coming from the simple closed curves $\delta_{1}, \ldots, \delta_{12}$ are represented by twelve unknots transverse to each fiber of the fibration $\Sigma_{2,0} \times S^{1} \rightarrow S^{1}$ and meeting a fiber at twelve points indicated by encircled numbers $1, \ldots, 12$ in Figure 21. Attaching a 2 -handle with framing -1 along any one of the twelve unknots together with four 3-handles and a 4-handle to $Y_{2}-v F$, we have a handle decomposition of the closed manifold $Y_{2}$.

By virtue of Theorem 3.1, we can also depict disjoint $4 g+4$ sections of the Lefschetz fibration $Y_{g} \rightarrow S^{2}$ in a Kirby diagram of $Y_{g}-v F$ for $g \geq 3$ in a similar way.

The following proposition implies that the largest possible number of disjoint (-1)sections of $X_{g} \rightarrow S^{2}$ is equal to $4 g+4$ for most $g$.

Proposition 4.7 If $g$ is not equal to $k^{2}+k-1$ for any positive integer $k$, then the Lefschetz fibration $X_{g} \rightarrow S^{2}$ cannot admit disjoint $4 g+5$ sections with selfintersection number -1 .

Proof Suppose that the Lefschetz fibration $X_{g} \rightarrow S^{2}$ admits disjoint $4 g+5$ sections $s_{1}, \ldots, s_{4 g+5}$ with self-intersection number -1 . The orientation of $S^{2}$ induces that of $S_{i}:=s_{i}\left(S^{2}\right)$ for $i=1, \ldots, 4 g+5$. We orient a regular fiber $F$ of $X_{g}$ so that it satisfies $[F] \cdot\left[S_{i}\right]=+1$ for $i=1, \ldots, 4 g+5$. Blowing down the $(-1)$-spheres $S_{1}, \ldots, S_{4 g+5}$ in $X_{g}$, we obtain a 4 -manifold $X^{\prime}$ and the image $F^{\prime}$ of $F$ under the projection $X_{g} \rightarrow X^{\prime}$. Since

$$
[F]=\left[F^{\prime}\right]-\left[S_{1}\right]-\cdots-\left[S_{4 g+5}\right] \quad \text { in } H_{2}\left(X_{g} ; \mathbb{Z}\right) \cong H_{2}\left(X^{\prime} ; \mathbb{Z}\right) \oplus(4 g+5) H_{2}\left(\overline{\mathbb{C P}}^{2} ; \mathbb{Z}\right)
$$

and $[F]^{2}=0$, we have $\left[F^{\prime}\right]^{2}=4 g+5$. On the other hand, $\left[F^{\prime}\right]^{2}$ must be the square of an integer because $\left[F^{\prime}\right]$ is a multiple of a generator of $H_{2}\left(X^{\prime} ; \mathbb{Z}\right) \cong \mathbb{Z}$. It is easy to see that $4 g+5$ is the square of an integer if and only if $g$ is equal to $k^{2}+k-1$ for some positive integer $k$. 


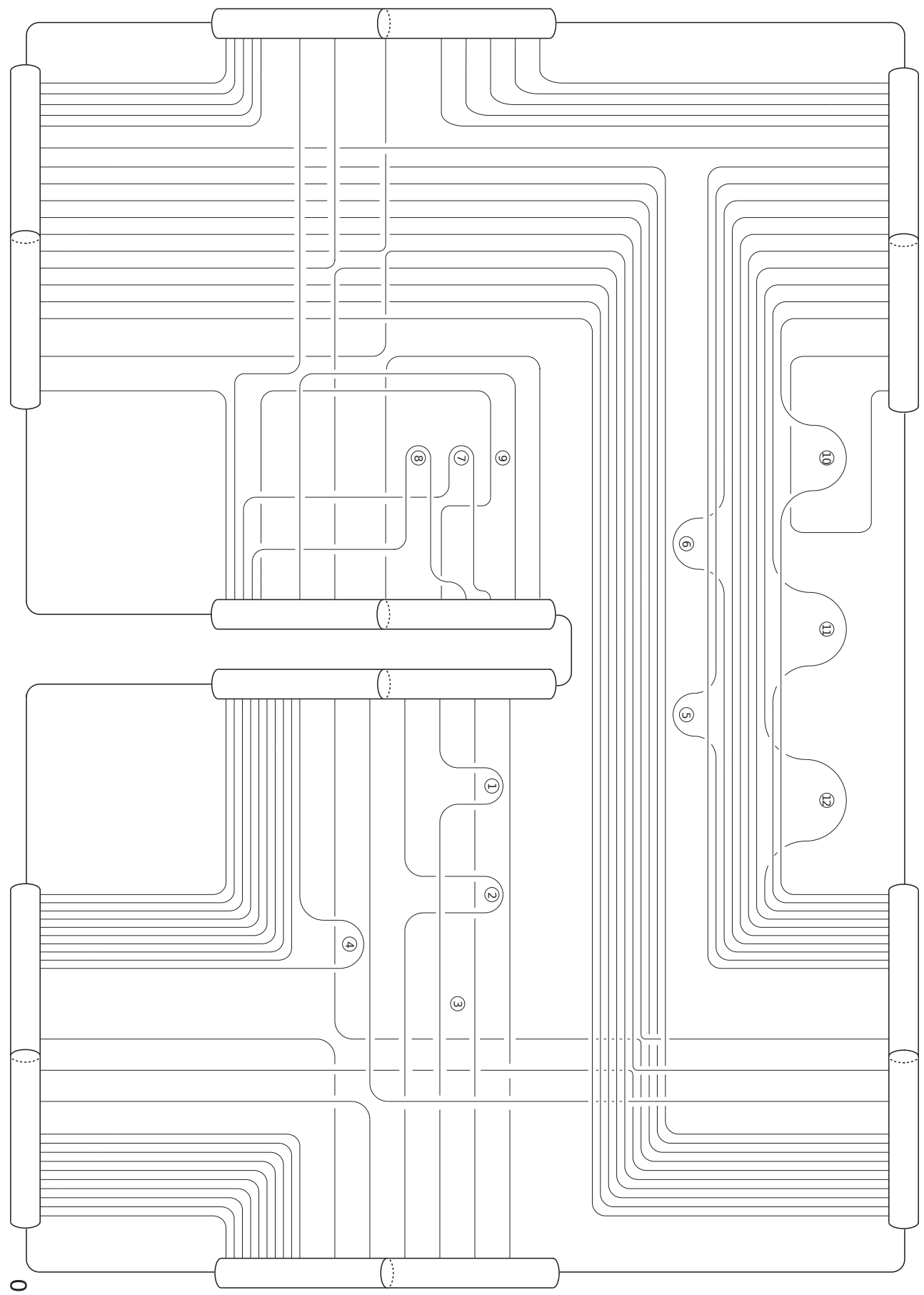

Figure 21: A Kirby diagram of $Y_{2}-v F$ 
Remark 4.8 Two generic degree $d$ curves in $\mathbb{C P}^{2}$ induce a Lefschetz pencil of genus $(d-1)(d-2) / 2$. Blowing up the base locus, we obtain a Lefschetz fibration $M_{d} \rightarrow S^{2}$ of the same genus. This fibration has $d^{2}$ sections with self-intersection number -1 and the total space $M_{d}$ is diffeomorphic to $\mathbb{C P}^{2} \# d^{2} \overline{\mathbb{C P}}^{2}$. It is well-known that the fibration $M_{3} \rightarrow S^{2}$ is isomorphic to $X_{1} \rightarrow S^{2}$, whereas the fibration $M_{d} \rightarrow S^{2}$ for $d \geq 4$ cannot be isomorphic to $X_{g} \rightarrow S^{2}$ for any $g$.

Acknowledgement The author is grateful to Professor Kazuhiro Konno for helpful comments on sections of fibrations on algebraic surfaces, and to Professor Yoshihisa Sato for helpful comments (Remark 4.8) on Lefschetz fibrations. And I wish to express my deep gratitude to my mentor, Hisaaki Endo.

\section{References}

[1] J Amorós, F Bogomolov, L Katzarkov, T Pantev, Symplectic Lefschetz fibrations with arbitrary fundamental groups, J. Differential Geom. 54 (2000) 489-545 MR1823313

[2] H Endo, M Korkmaz, D Kotschick, B Ozbagci, A Stipsicz, Commutators, Lefschetz fibrations and the signatures of surface bundles, Topology 41 (2002) 961-977 MR1923994

[3] B Farb, D Margalit, A primer on mapping class groups, Princeton Math. Series 49, Princeton Univ. Press (2012) MR2850125

[4] R E Gompf, A I Stipsicz, 4-manifolds and Kirby calculus, Graduate Studies in Mathematics 20, American Mathematical Society (1999) MR1707327

[5] T Ito, Splitting of singular fibers in certain holomorphic fibrations, J. Math. Sci. Univ. Tokyo 9 (2002) 425-480 MR1930415

[6] S Kitagawa, K Konno, Fibred rational surfaces with extremal Mordell-Weil lattices, Math. Z. 251 (2005) 179-204 MR2176471

[7] M Korkmaz, B Ozbagci, On sections of elliptic fibrations, Michigan Math. J. 56 (2008) 77-87 MR2433657

[8] Y Matsumoto, Lefschetz fibrations of genus two-a topological approach, from: "Topology and Teichmüller spaces", (S Kojima, Y Matsumoto, K Saito, M Seppälä, editors), World Sci. Publ., River Edge, NJ (1996) 123-148 MR1659687

[9] S Ç Onaran, On sections of genus two Lefschetz fibrations, Pacific J. Math. 248 (2010) 203-216 MR2734172

[10] M-H Saitō, K-I Sakakibara, On Mordell-Weil lattices of higher genus fibrations on rational surfaces, J. Math. Kyoto Univ. 34 (1994) 859-871 MR1311624 
[11] I Smith, Geometric monodromy and the hyperbolic disc, Q. J. Math. 52 (2001) 217-228 MR1838364

Department of Mathematics, Graduate School of Science

Osaka University, Osaka 560-0043, Japan

qqx33r5d@almond.ocn.ne.jp

Received: 6 March 2012 Revised: 10 August 2012 